\title{
I ROMANISMI NELLA TERMINOLOGIA VITICOLA DELL'ISTRIA SLOVENA
}

Il presente saggio trae spunto da un attento riesame del materiale che ho raccolto ed analizzato per la mia tesi di dottorato di ricerca dal titolo Poljedelska in vinogradniška terminologija v govorih Slovenske Istre (La terminologia agricola e viticola nelle parlate dell'Istria slovena), tesi che ho discusso presso la Facoltà di Filosofia di Lubiana il 14 gennaio 1994, relatore il prof. Tine Logar, correlatore la prof. Alenka Šivic-Dular. L'opera si inserisce nel filone di ricerche lessicali orientate a riscrivere la storia linguistica di questa regione così complessa per le innumerevoli vicende storiche e per i molteplici contatti linguistici tuttora fertili.

Con l'intento di individuare i romanismi presenti nelle parlate slovene dell'Istria ho raccolto negli anni 1988-90 cospicuo materiale secondo un questionario di $97.8 \mathrm{do-}$ mande che ho compilato, adattandolo alle esigenze economiche, culturali e nazionali dell'Istria, sulla base dei questionari dell'ALI, dell'ASLEF, dello SLA e con l'aggiunta di alcune domande personali contrassegnate con la sigla RC. Ho svolto le inchieste dialettali nei seguenti 10 punti che coprono in modo uniforme l'Istria slovena: 1 Malija, 2 Padna, 3 Krkavče, 4 Gažon, 5 Šmarje, 6 Koštabona, 7 Pomjan, 8 Boršt. 9 Marezige, 10 Trebeše.

La prima parte dell'opera riporta il materiale raccolto diviso in 7 sezioni secondo le attività agricole prevalenti:

I Gli attrezzi agricoli, il raccolto, il lavoro nei campi e nella stalla;

II Il lavoro nell'orto e nel frutteto;

III La viticoltura;

IV Alcune piante ed alberi nonché le attività connesse;

V L'allevamento del bestiame;

VI L'apicoltura;

VII La coltivazione dell'ulivo e la produzione dell'olio.

La seconda parte dell'opera è la trattazione linguistica del materiale raccolto. I 554 romanismi, analizzati anche dal punto di vista etimologico, vengono divisi in 5 strati: i latinismi, i friulanismi, i venetismi, gli istrianismi, i triestinismi e gli italianismi. Dalla sezione riguardante la viticoltura riporto nel seguente elenco i romanismi piú significativi: 
458. Ceppo di vite selvatica; Q. RC:

a) 6. ra'parja

L'unica connessione possibile della voce istr.slov. è quella con il bis. ripària bot. 'vite selvatica, americana' (Domini 379), derivato dall'agg. lat. ripariu(m), a sua volta dal lat. rīpa(m) 'riva', a cui corrisponde l'it.ant. ripa 'id.', it. riva (DELI 4, 1085; REW 7328). Nella toponomastica istr. (Medulino) Ripa è attestato nel 1454 (Rosamani 883).

b) 6. rə'pẹšte

Il termine istr.slov. è d'incerta interpretazione: anche l'unica ipotesi, che si tratti di un'incrocio dell'istr.slov. ra'parja (per l'et. v. 458a) con una voce ignota, non è convincente.

459. Tralcio; Q. ALI 3940:

9. špa'ruọn

La voce istr.slov., affine allo stir.sorient. šparôn / šperôn 'tralcio di vite tagliato in cinque e più butti' (Plet. II 641 e 642), deriva dal triest. (anche mugl.) ed istr. (C., Pir., Mt., Cf., D.) spiron 'penzolo d'uva' (DDM 159; GDDT 667; Rosamani 1074; Semi 293), variante, munita di s- rafforzativo-espressiva, dell' istr. (Pir.) piron (de ua) 'id.' (GDDT ibid.; Rosamani 797), a cui corrisponde il triest. e ven. piron 'forchetta'; cfr. anche il vic. pierón, rover. e trent. piróm dello stesso significato, ver. birón 'perno', venez. pirón 'forchetta; perno' (Prati EV 132) ed it.ant. piróne 'cavicchio' (DEI IV 2947). Dal gr.biz. p(e)iròuni, continuatore del gr.ant. perònion 'piccolo cavicchio, perno' (GDDT ibid.; REW 6366) e non da un * pirio 'succhiello' di origine gr. come propone il Prati (Prati ibid.).

460. Tralciaia (piu tralci intrecciati); Q. ALI 3941:

a) 6. pa'rẹt

Dall'istr. (C., Is., Mt., P.) parè 'filare di viti; parete; (Rosamani 736; Semi 275; Vascotto 198) / mugg. pareo (Rosamani 737) / triest. paredo (GDDT 435), a cui corrisponde il friul. paréit / parêt 'parete d'assi' (NP 701 seg.), bis. paredo 'parete divisoria' (Domini 322), ven. (anche venez.) paré 'tramezzo' (Boerio 472; Prati EV 120) ovvero l'it. paréte 'id.'. In ultima analisi si tratta di un prestito dal lat. parl. * parēte(m) 'parete' per il class. pariete(m) (DELI 4, 880 seg.; REW 6242).

b) 9. špa'ruọni (pl.) (per l'et. v. 459) 
464. Palo da vite; Q. ALI 3945:

a) 1. fər'kada; 2. fər'kada; 3. fər'kådå; 4. fər'kada; 5. fər'kådå; 6. fər'kada; 7 . fər'kåda; 8. fər'kada

Il termine istr.slov. concorda, anche semanticamente, con istr. (C.) forcada 'legno biforcato ad un'estremità che serve a sostenere la corda del bucato' (Rosamani 393; Semi 255) che si può confrontare anche con venez. forcada 'forcata; specie di asta di legno che usano le lavandaie quando distendono sulle corde o funi le biancherie lavate' (Boerio 281) e friul. forciàs 'forcone; pertica biforcuta per sostegno di tralci o di corde tese' (NP 334) nonché friul. forciàde 'forcata' (NP ibid.) ed it.ant. forcata 'id.' (DEI III 1686), a sua volta da it. fórca (DEI III 1685), dal lat. fürca, relitto mediterraneo (REW 3593).

b) 3. ka'nẹlå; 4. ka'nẹla; 7. kə'nẹla; 8 . ka'nẹla; 2 . kə' niẹla; 5. kə'nẹlå

Dal triest. ed istr. (C., V., Is.) canèla 'piccola canna; cannella (della botte)' (GDDT 121 e 871; Cernecca 28; Rosamani 159; Vascotto 70) che concorda, anche semanticamente, con friul. canèle (DESF I 303; FPF I 196; NP 97), bis. canela (Domini 80), mugl. * kanéla (DDM 69), bellun. kanèla (Tomasi 87), ven. (anche venez.) canèla (Boerio 127; Durante 72) nonché it. cannèlla, dim. di it. cànna (DEI I 722; DELI 1, 196). Dal lat. cănna(m) 'canna', a sua volta dal gr. kánna 'id.' di origine assirobabilonese (REW 1597).

c) 5. 'lånčå

La voce istr.slov., che concorda con lo slov. lânča 'lancia' (Bezlaj II 123; Mende RLS 87; Plet. I 499), rieccheggia l'it. lett. lància 'id.' (DEI II 2160) nonché l' istr. (C., Is., Pir., V., Mt.) lansa (Cernecca 57; Rosamani 525; Vascotto 149), triest. ed istr. (Mt., F.) lanzia (Rosamani ibid.), friul. lance (NP 500) / lànze (NP 501), venez. lanza (Boerio 359), termini d'area romanza occid. derivati dal lat. lancea 'lancia' (REW 4878).

465. Vigna, vigneto; Q. ALI 3946:

a) 1. 'vińa; 2. 'vińa; 3. 'vińå; 4. 'vińa; 5. 'vinå; 6. 'vińa; 7. 'vińa; 8. 'vińa; 9. 'vińå; 10. 'vina

Voce mutuata dal triest. ed istr. (P.) vigna 'vigna' (GDDT 784; Rosamani 1221), cfr. anche rov. $\mathbf{v}(\mathbf{u})$ éigna (Rosamani 1238), che convergono con il friul. vìgne (NP 1275), bis. vigna (anche 'uliveto') (Domini 536), padov., venez., valsug., ver., rover., trent. vigna 'vite; (venez.) 'vigna' (Boerio 793; Prati EV 201) ed it.lett. vigna dello stesso significato (DEI V 4051), esiti del lat. vinea(m), parl. * vinja, propriamente un agg. sostantivato vineu(m) 'appartenente al vino' (DELI 5, 1438; REW 9350). 
b) 2. b'rajda

Il termine istr.slov., che risponde allo slov. cars. brájda 'appezzamento di un campo coltivato a viti' (Bezlaj I 37; Mende RLS 49; Plet. I 49) e scr. brâjda 'vigneto' (Bezlaj ibid.), è un romanismo: si accorda con istr. bràida 'vigna' (Rosamani 112; Samani 37) che trova riscontro anche nel triest. bràida 'prato della periferia della città' (GDDT 90), terg. bràida 'vigneto' (Rosamani ibid.), mugl. bráida / brália 'terreno arativo in piano' (DDM 16), bis. bràida 'poderetto recintato vicino casa' (Domini 58), friul. bràide 'id.' (DESF I 260; NP 71), venez. bràida 'id.' (Boerio 97), emil. braia, bresc. breda 'poderetto' (DEI I 587). Voce di area it. sett. derivata dal long. braida 'campo pianeggiante, pianura' (REW 1266).

466. Filare di viti nella vigna; Q. ALI 3947:

a) 1. lə'rańa

Dall'istr. (Pir.) liragna 'spazio di terreno tra due filari di viti largo un doppio solco' (Rosamani 545), voce isolata di origine romanza: dal lat. lira 'porca', donde anche l'it.ant. lira 'grado, posto' (DEI III 2246).

b) 4. pə'rẹt; 5. pa'rẹt; 6. pa'rẹt; 8. pa'rẹt; 9. pə'riẹt 'tərt; 10. pa'rẹt (per l'et. v. 460a)

467. Pergola; Q. ALI 3948:

1. 'peryola; 2. 'piẹryola; 3. 'peryolå; 4. 'peryola; 5. 'peryolå; 6. 'peryola;

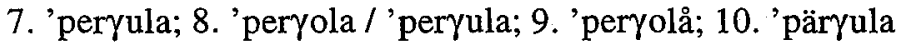

Il termine istr.slov., affine, anche dal punto di vista semantico, allo slov. goriz. e cars. piéryla 'tipo d'uva' (Mende RLS 103; Štrekelj, Görz. 436) e chiogg. ùga pèrgola (GDDT 450), deriva dal triest. ed istr. (C., Is., V.) pèrgola 'pergola' (GDDT ibid.; Cernecca 82; Rosamani 762; Vascotto 206) che trova vari raffronti nell' area marginale ven.: cfr. ven. (anche venez.) e bellun. pèrgola (Boerio 492; Durante 368; Tomasi 141), mugl. pérgula (DDM 118), bis. pèrgula (Domini 332) ovvero it. pèrgola. Dal lat. pĕr. gula(m) 'ballatoio' (DEI IV 2850; DELI 4, 908; REW 6413).

468. Brenta; Q. ALI 3949:

a) 1. brən'tẹla; 2. brən'tiẹla; 3. brən'tẹlå; 4. brən'tẹla; 5. brən'tẹlå; 6. brən'tẹla; 7 . brən'tela; 8 . brən'tela; 9 . brən'tielå; 10 . bran'tela

Termine mutuato dal triest. ed istr. (C., P.) brentela / (Is.) brentèl 'bigonciolo a un manico' (GDDT 92; Rosamani 116; Vascotto 59) che concordano anche semanticamente con il mugl. brentiéla / brantiéla (DDM 17), friul. brentèle (DESF I 265; NP 74), bis. brentel (Domini 60), bellun. brentèl (Tomasi 43), vic. brentèla 'cassone', ver. 
brentèla 'tinozza' (Prati EV 25), forme dim. di brenta, voce it. sett. diffusa fino alla Svizzera e probabilmente di origine preromanza.

\section{b) 1 . bran'tẹlca}

Trattasi di diminutivo romanzo ampliato con ulteriore suffisso dim. slov. -ica / -ca (per l'et. v. 468a).

469. Grappolo; Q. ALI 1976:

\section{1. rə’ćela}

Dall'istr. (Is., V., B.) ricèla 'racimolo d'uva' (Rosamani 878; Vascotto 237) / (D.) riccièla (Dalla Zonca 220) / (Is.; anche triest.) rincèla (GDDT 525; Rosamani 882; Vascotto ibid.) che presuppongono un caso di etimo 'multiplo' (GDDT ibid.): è da accostare, anche semanticamente, il ver. récia d'ua, bellun. régia d'ua (Prati EV 142), venez. reciotín de ua (Boerio 559; Prati ibid.), ven. récio (Durante 419) / recéto / reciéto (Durante 418), ven.dalm. recèl (Miotto 168); dal ven. recia 'orecchio' (Prati ibid.), a sua volta dal lat. aurǐcula 'id.' (REW 793). Però il termine dialettale triest. ed istr. rincèla potrebbe essere un interessante friulanismo se si considera il friul. rincièle, dim. del friul. rìncie 'fibbia' (NP 879) di origine germ. (dal germ. hringa 'fibbia, anello'). Sennonché il friul. usa anche la forma ricièla 'fibbia' (NP 872) che è indubbiamente il dim. del ven. recia. Il dilemma è forse risolvibile supponendo che all'epoca di formazione dei diminutivi i due termini, reciela e rinciela, si erano avvicinati fra di loro per motivi semantici e che a un certo punto il secondo, per influsso del primo, abbia perduto la nasale (GDDT ibid.).

470. Graspo; Q. ALI 3950:

a) 1. 'rašpa; 2. 'rašpa; 3. 'råšpå; 6. 'rašpa; 8. $\gamma$ 'råšpa

La voce istr.slov., che risponde anche al res. grâsp 'grappolo' (Mende RLS 71; Plet. I 245), deriva dall' istr. raspa / graspa 'graspo' (Rosamani 860 e 452; Vascotto 129) che ha varie concordanze nell'area marginale: cfr. il triest. graspa 'vinacce' (GDDT 278) / graspo 'grappolo' (GDDT ibid.), friul. grasp 'id.' (NP 401), bis. rasp / grasp 'grappolo, graspo' (Domini 368 e 211), mugl. ráspi pl. 'vinacce' (DDM 132), vic., ver., venez. graspa 'graspo' (Boerio 315; Prati EV 78) / padov., ver., bellun., trev., venez. graspe pl. 'vinacce' (Boerio ibid.; Durante 211; Prati ibid.), ven. e ven.dalm. graspo 'grappolo' (Miotto 91; Prati ibid.) affini all' it. gràspo 'raspo' (DEI III 1862; DELI 2, 517) / ràspo (DEI V 3210; DELI 4, 1034). L'it. graspo (con g- protetica) sarebbe il risultato dell'incrocio tra l'it. raspo ed it. grappolo (DEI II 1862), mentre l'it. ràspo è deverbale di it. raspar 'grattare via', a sua volta dal germ. raspōnn 'id.' (DEI V 3210; REW 7077). 


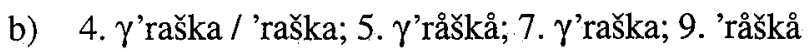

Voce mutuata dall'istr. (C.) rasca 'graspo' (Rosamani 859; Semi 282) connesso con il friul. ràs'cie 'grapppoletto' (NP 850), mugl. rásča 'racimolo' (DDM 132), bis. ras'cia 'id.' (Domini 368), triest. dim. ras'cela 'id.' (GDDT 511). Il termine istr. è deverbale di istr. (C.) rascàr 'grattare', a sua volta dal lat. * rasicāre 'id.' (REW 7074).

473. Penzolo; Q. ALI 3953:

1. špə'rọn; 2. špə'ruọn; 3. špə'rọn; 4. špə'rọn; 5. špə'rọn; 6. špa'rọn; 7. špə'rọn

8. špa'run / špe'rọn; 9. špa'ruọn; 10. špə'rọn (per l'et. v. 459)

475. Tinella; Q. ALI 3955:

a) 8. ti'nẹlca

Il termine istr.slov., ampliato con l'agggiunta del suffisso dim. slov., deriva dall'istr. (C.) tinèla 'piccolo tino' (Rosamani 1155) / (R.) tiniela 'tino alto $1,50 \mathrm{~m}$ ' (Rosamani ibid.) che convergono con il friul. tinèle 'recipiente per conservare la farina' (NP 1191), bis. tinela 'tino' (Domini 508), venez. tinela / tinèta 'tinozza' (Boerio 749) ed it. ant. tinèllo 'piccolo tino' (DEI V 3794) / tinella 'id.', forme dim. dell'it.ant. tina 'piccolo tino', a sua volta dal lat. tīna(m) 'sorta di bottiglia da vino' (DEI V 3793; REW 8741 1.).

b) 10. ti'nåco

Mutuato dal triest. ed istr. (A., Ch.) tinazo 'tino' (GDDT 735; Rosamani 1155) / (C., D., R.) tinaso (Rosamani ibid.) con vari raffronti nella prossima area friul.-ven.: cfr. anche il triest. tinaz 'id.' (GDDT ibid.; Rosamani ibid.), friul. tinàz 'tino principalmente per la pigiatura dell'uva' (NP 1190), bis. tinaz 'tino di grandi dimensioni' (Domini 508), bellun. tinàz 'sorta di tino che si mette sul carro per raccogliervi l'uva quando si vendemmia' (Tomasi 198), venez. tinazzo 'vaso per farvi bollire il mosto' (Boerio 749), ven. tinàsso / tinàzo 'id.' (Durante 619). Le voci sopraccitate sono formazioni aumentative dell' italianismo tìno, a sua volta dal lat. tardo * tinu(m) (DELI 5 , 1340; REW 8741 2.).

477. Strettoio (torchio) da vino; Q. ALI 3957:

a) 2. št'ruk; 3. št'ruko; 4. št'ruk; 7. š'truk; 8. št'ruk; 10. št'ruk

Dall'istr. (Is., Pir.) strùco 'torchio per pigiare le vinacce' (Rosamani 1111; Vascotto 306) affine al triest. struco 'estratto' (GDDT 699), friul. strùc 'succo che si ottiene dalla spremitura' (NP 1138), ven. (anche venez.) e bellun. struco 'id.' (Boerio 718; Durante 591; Prati EV 181; Tomasi 193). I termini sopraccitati sono forme deverbali da strucar 'spremere', voce di area it. sett. (anche lomb.) per la quale vengono pro- 
posti vari etimi: 1) dal lat. * trūdĭcāre 'spingere'; 2) dal lat. extŏrquere 'estorcere'; 3) da un onomatopeico * trucch (Prati ibid.); 4) considerata l'esistenza di forme come trucar 'battere, colpire' è preferibile la derivazione dal got. thruk-s (ted. Druck) 'colpo' (DEI V 3661).

\section{b) 5. p'rẹšå}

La voce istr.slov., che concorda anche con lo slov. préša 'torchio per l'uva' (Plet. II 279), deriva dal triest. presa 'pressa' (GDDT 491) che nell' accezione di 'fretta' ha vari raffronti: cfr. l'istr. pressa (Rosamani 828; Samani 139), ven. (anche venez.) prèssa (Bernardi 321; Boerio 533; Durante 396; Prati EV 137), poles. e ver. prèssia (Prati ibid.), ven.dalm. prèsa (da cui cr.dalm. prǐša) (Miotto 161), friul. prèsse (NP 807), mugl. présa (DDM 355), bis. pressa (Domini 355) ovvero it. prèssa anche 'torchio', deverbale di pressare 'premere forte', a sua volta dal lat. pressāre 'id.'. Nel significato 'macchina per comprimere' è un calco sul fr. presse (DEI IV 3070; DELI 4, 976).

481. Vigna giovane; Q. ALI 3961:

1. 'vińa 'nova; 2. m'lada 'vińa ; 3. m'lådå 'vińå; 4. m'låda 'vińa ; 5. 'vińå m'lådå; 6. m'låda 'vińa; 7. 'vińa m'låda; 8. m'låda 'vińa; 10. 'vińa m'låda (per l'et. v. 465a)

482. Vignaiolo; Q. ALI 3962:

2. pərdə'lavəc 'vina

Il termine istr.slov., che trova riscontro anche nello slov. lett. vínọ dello stesso significato (Plet. II 770), è un antico prestito romanzo: dal lat. vīnum 'id.', cfr.. it. vìno (DELI 5, 1440; REW 9356; Rocchi 9356).

483. ROŽJE; sarmento; Q. ALI 3963:

a) 1. sər'mẹnta; 2. sərə'miẹnta; 3. sərə'mẹntå; 4. sərə'menta; 5. səra'mẹtå; 6. sara'mẹnta; 7. sər'mẹta; 9. sara' mẹntå

Dall'istr. (C., Is., Pt., Mt.) bot. sarmenta 'sarmento' (Rosamani 935; Vascotto 251) che ritorna anche nel mugl. pl. sarmiénti / sermiénti 'id.' (DDM 142) ovvero it.lett. sarménto (per l'et. v. 483b).

b) 8. sarə'mento

Voce mutuata dall'istr. (C., Pir.) sarmento 'sarmento' (Rosamani 935; Semi 286) / (R.) sarmaènto (Rosamani 934 seg.) che concorda con l'it. sarménto 'id.', dal lat. sar- 
mĕntu(m) 'rami secchi', a sua volta dal lat. sărpere 'potare' di origine ie. (DEI V 3343; DELI 5, 1130; REW 7609).

486. Coltura a terrazzi (vitigno a terrazzi); Q. ALI 4919:

a) 1. 'piẹce (pl.)

Dall'istr. (Pir.) pieza 'terrazza; appezzamento di terreno coltivato a terrazze' (Rosamani 787) / (Pir., B.) piesa 'id.' (Rosamani 786) che convergono anche con il venez. pezza de tera 'pezzo di terra; la parte di uno o più campi' (Boerio 501) ed it. pèzza 'tratto di terra coltivata' (pecia in un documento venez. del 1253), dal lat.parl. *pĕttia(m) 'pezzo', voce di origine celtica (DEI IV 2888; DELI 4, 917; REW 6450).

b) 1. 'banda

Il termine istr.slov., affine nel secondo significato di 'sbarre del carro stretto' al cars. pl. bandə 'id.' (SDLA-Ts 448), è di origine ven.: cfr. venez., ven., triest., bellun. banda 'banda, lato' (Boerio 61; Durante 22; GDDT 53; Rosamani 63; Tomasi 33), friul. bànde f. 'lato, parte' (NP 35), a Pieris bánde del kar 'sbarre del carro stretto' (TAF 337). Dal got. bandwja 'segno', forse attraverso il prov. banda 'parte', cfr. it. banda 'striscia di tessuto, lista, fascia' (DEI I 424; DESF I 153).

\section{c) 2. 'vińa nə p'jante}

Il termine istr.slov. è indubbiamente un italianismo: da it. pianta (DEI IV 2894), attestato in quasi tutti i dialetti it. sett.: cfr. triest., istr., venez. pianta (Boerio 503; GDDT 458; Rosamani 777). Tuttavia lo sviluppo semantico 'filare di alberi o di viti', che si riscontra nella voce istr.slov., è parallelo al bis. pianta (Domini 337; Rosamani ibid.), friul. plànte (NP 776), da cui cars. planta 'id.' (SDLA-Ts 492), nonché bellun. pianta (Tomasi 142). Dal lat. planta 'stelo o pollone', deverbale da lat. plantāre 'piantare' (REW 6575).

d) 4. 'vińa (per l'et. v. 465a) nə pə'rẹde (per l'et. v. 460a); 10. 'vińa na pa'rẹt

e) 6. tə'råca

Dal triest. teraza, -o 'terrazza' (GDDT 730; Rosamani 1147) / istr. (C., V., Pl.) terasa (Cernecca 113; Rosamani ibid.) che vanno con friul. teràzze (NP 1184), bis. teraza (Domini 504), ven. teràssa /teràza (Durante 617), venez. terazza (Boerio 744) nonché it. teràzza, a sua volta dal fr. terasse ovvero prov. terrassa; cfr. lat.med. terracia, terrassia (DEI V 3765; DELI 5, 1331).

f) 5. 'vińå nə 'påšná; 9. 'vińå nə 'påštin 
Voce istr.slov., affine anche al triest.slov. pášten / pášen 'terreno recintato e coltivato a vigneto' (Mende RLS 102; Plet. II 13), derivata dal triest. pàstino / pàsteno / pàstano 'campo coltivato a terrazze' (GDDT 439; Rosamani 746) ovvero istr. (C., Is., Pir.) pàsteno 'id.' (Rosamani ibid.; Vascotto 202) che risponde anche al mugl. pásten 'campo vitato' (DDM 116). I termini sopraccitati sono forme deverbali del triest. ed istr. pastinar, continuatore di lat. pastinare 'piantar viti' (REW 6276).

\section{g) 8. 'vińa nə pašta'nẹl}

La voce istr.slov., sebbene isolata, è indubbiamente una forma dim. del triest. pàstano (per l'et. v. 486f). L'unica connessione possibile è con l'istr. (Is.) pastenàl 'coltivazione di sole cucurbitacee' (Vascotto 201), a sua volta dim. di istr. pasteno (v. $486 f)$.

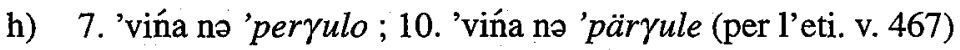

i) 4. vińa nə b'rajde (per l'et. v. 465b)

490. Vimini, vermena di salcio; Q. ALI 3967:

1. 'vẹnka; 2. 'viẹnka; 3. 'vẹnkå; 4. 'vẹnka; 5. 'vẹnkå; 6. 'vẹnka

La voce istr.slov. è la femminizzazione del triest. ed istr. (C., Is., B., V., F.) bot. vénco 'vinco' (GDDT 778; Cernecca 119; Rosamani 1209; Semi 300; Vascotto 330) / (Ch.) vencio (Rosamani ibid.) / (Lgr.) vincio (Rosamani 1225) che concordano con friul. vènc (FPF II 560 seg.; NP 1263), bis. venc (Domini 532), mugl. viénk / venk (DDM 180), bellun. vénk (Tomasi 206), trev. pl. vénchi (Bernardi 457), venez. venchio (Boerio 784) ovvero it.ant. vénco (DEI V 4008) ed it.lett. vìnco, da un lat.parl. *vincu(m) 'legame di salcio', a sua volta dal lat. vinculum 'legame' (DEI V 4058; DELI 5, 1439; REW 9342).

492. Mettere i pali nel vitigno; Q. RC:

a) 1. impa'lavət; 2. im'pảlàt; 5. im'pảlat; 6. impa'låvat; 8. impa'lirat; 9. impa'låvat; 10. nə'pålat

Gli infiniti istr.slov. sono, con l'aggiunta di vari prefissi e suffissi slov., degli adattamenti del venez. impalàr 'impalare' (Boerio $326 \mathrm{seg}$.) che trova riscontro nel ptc.pass. triest. ed istr. (C., Is.) impalà 'eretto, impalato'(GDDT 294; Rosamani 477; Semi 261; Vascotto 135), nel friul. impalâ 'impalare' (NP 425) nonché it.lett. impalare 'id.' (DEI III 1953), derivato da it. palo, a sua volta dal lat. palus 'palo' (REW 6182).

b) 4. s'tavit far'kade ; 7. s'taúlät far'kåde (per l'et. v. 464a) 
a) 1. 'čəmət; 2. po'čəmat; 3. 'čẹmat; 6. 'čemat; 7. 'čemàt

Adattamento del triest. ed istr. (F.) zimar 'cimare' (GDDT 810; Rosamani 1257; Samani 213) ovvero istr. (Is., Pir., B., A., Cf.) simar 'id.' (Rosamani 1030; Vascotto 279) / (Pir., V.) simâ (Cernecca 103; Rosamani 1030) che ha vari riscontri dello stesso significato nell' area prossima: cfr. friul. cimâ (DESF II 393; NP 150), bis. zimàr (Domini 553), ven. zimare (Durante 663), venez. cimàr (Boerio 171), ven.dalm. zimàr (Miotto 224) e da ultimo it. cimàre (DEI II 937; DELI 1, 237), derivato da it. cima 'vetta'.

b) 4. 'mọndət

Dall'istr. (Pir.) mondâ 'mondare (il grano, gli alberi, le viti) (Rosamani 641) / (D.) mondà (Dalla Zonca 192) / (A., Mt.) mondar (Rosamani ibid.) che concordano, anche dal punto di vista semantico, con friul. mondâ (NP 611), mugl. mundár (DDM 102) nonché it. mondare (DEI IV 2494). Dal lat. tardo mŭndāre 'pulire' (REW 5744).

496. Aspergere; Q. RC:

1. 'dajət vardə'rame ; 3. 'dåjət vardə'råme ; 4. 'däjət varda'rame ; 5 . 'dajjat

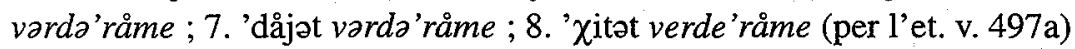

497. Solfato di rame; Q. RC:

a) 1. vərdə'rame; 2. vərdə'rame; 3. vərdə'råme; 4. vərdə'rame; 5 . vərdə'råme; 6. vərdə'rame; 7. vərdə'råme; 8. verde'råme 9. vərde'råme; 10. vərdə'råm / vərdə'råme

Dall'istr. (Is.) verderàme 'miscela di calce e di solfato di rame' (Vascotto 330) che ha echi nei repertori friul. e ven. limitrofi: cfr. dello stesso significato il bis. verderame (Domini 533), friul. verderàm (NP 1266), ven.dalm. verderàme (Miotto 217), venez. verderàme (Boerio 787) nonché it. verderàme, termine composto da it. verde ed it. rame, poiché il solfato di rame costituisce la patina 'verde' degli oggetti di 'rame' lasciati all'umido (DEI V 4018; DELI 5, 1425).

\section{b) 9. vədər'jọl}

La voce istr.slov., affine, anche semanticamente, allo slov. cars. vədərjol, può essere posta in connessione con il ven. dalm. vedriòl 'solfato di rame' (Miotto 217) ed it. vetri(u)òlo 'solfato di metalli pesanti', voce dotta che riconduce al lat. tardo vitreŏlu(m) 'di vetro' (dim. del sost. vĭtriu(m) 'vetro'), poi sostantivato in vitriŏla(m) 'vetriolo azzurro o verde; solfato di ferro o di rame' (DEI V 4042; DELI 5, 1433). 
500. Mantice; Q. RC:

a) 4: 'fọla; 5 .'fọla

Vóce mutuata dal triest. ed istr. (C., Is., Pir., B., V.) fola 'mantíce' (GDDT 241; Cernecca 47; Rosamani 389; Semi 255; Vascotto 114) che converge, anche semanticamente, con il friul. fòle (NP 330), padov., venez., trev., bellun. fòla (Boerio 278; Prati EV 66; Tomasi 62). Dal lat. fŏllis 'sacco di cuoio, mantice' (REW 3422).

b) 1. 'fọlo

Dal triest. ed istr. (C., Is., P., B., O., Pl., F.) folo 'mantice' (GDDT 241; Rosamani 389; Samani 72; Semi 255; Vascotto 114) / (V., D.) fôl 'soffietto (per le viti)' (Cernecca 47; Dalla Zonca 127) / (D.) fòllo 'id.' (Dalla Zonca ibid.) con vari raffronti nell' area marginale: cfr. dello stesso significato il mugl. fólo (DDM 46), bis. fól (Domini 183), friul. fòl (NP 329), vic., ver., padov., venez., trev., ven.dalm. fòlo (Boerio 278; Durante 176; Miotto 82; Prati EV 66). Per l'et. v. 500a.

\section{c) 5. 'pọmpå; 8. 'pumpa; 9. 'puọmpå}

Mentre l'istr.slov. pumpa è affine allo slov. pūmpa 'pompa aspirante', che il Pleteršnik riconduce al ted. Pumpe (Plet. II 363), il termine istr.slov. pompa deriva dall'istr. (R., V.) agr. pompa 'irroratrice per le viti' (Cernecca 85; Rosamani 813) / (C.) ponpa (Semi 278) con vari raffronti dello stesso significato nell'area marginale: cfr. il friul. pòmpe (NP 794), bis. ponpa (Domini 350), venez. pompa (Boerio 520) nonché it. pómpa, dal fr. pompe di origine onomatopeica (DEI IV 3011; DELI 4, 954).

502. Recipiente in generale; Q. RC:

a) 1. 'važo

Dal triest. ed istr. (Is., V.) vaço 'vaso, recipiente' (GDDT 775; Cernecca 118; Rosamani 1203; Vascotto 328) che concorda, anche semanticamente, con il venez. vaso (Boerio 780), friul. vâs (NP 1258), mugl. vas (DDM 177), bis. vaço 'recipiente per olio' (Domini 530) nonché it. vàso 'recipiente', a sua volta dal lat.volg. vāsu(m), tratto dal pl. vāsa del class. vās, vāsis (DEI V 3994; DELI 5, 1414 seg.; REW 9161).

b) 1. yo'lida

Il termine istr.slov., affine allo slov. golída 'recipiente di legno per mungere' e scr. gòlida 'id.' (Bezlaj I 158 seg,; Mende RLS 70; Plet. I 227), deriva dall'istr.rom. (D.) galìda 'mastello' (Dalla Zonca 135; Rosamani 418) / (V., Gall.) galéda 'mastello, recipiente di legno a doghe' (Cernecca 51; Rosamani 418) che vanno anche con (Pir.) galedo 'brocchetto per il vino' (Rosamani 418) / (D.) galedel 'secchio per mungere' (Rosamani ibid.), friul. gialède 'id.'(NP 377), terg. pl. gulìdis 'scodelle' (Rosamani 
464), rover. gale(d)a, trent. galeda 'antica misura per l'olio' (Prati EV:71), istr.. (Cherso- sec. XV) galita 'misura di vino' (Rosamani 419), valtell. galeda 'bigonciolo per il vino', (Prati ibid.) ovyero it.ant. dial. galléta 'secchio' (DEI III 1752). Dal lat.mediev. gallēta 'id.' (DEI ibid.; Prati ibid.).

\section{c) 1. štə'ńak}

La voce istr.slov., affine anche al cars. štenják 'secchio per attingere l'acqua' (Plet. II 644), deriva dal triest. ed istr. (Is., R., V.) stagnaco 'secchio' (GDDT 677; Cernecca 108; Rosamani 1086; Vascotto 299) che va, anche semanticamente, con terg. stagnac (Rosamani ibid.), mugl. stańák (DDM 161), friul. pl. stagnàcs 'secchie di latte' (NP 1107), bis. stagnaco (Domini 471), ven.dalm. stagnàco (Miotto 200). Per dissimilazione di -ato > -aco dall'it. stagnato o stagnata 'recipiente di stagno' (DEI V 3614), a sua volta da it. stagno, dal lat. stagnum 'stagno' (DEI ibid.; REW 8217b).

\section{d) 4. 'kärəto; 5. 'kärto; 6. 'karatu; 10. 'kärato}

Nonostante il termine istr.slov. sia isolato, è indubbiamente connesso con il triest. ed istr. (Is., V., D., F.) dim. caratèl 'caratello' (GDDT 130; Cernecca 29; Dalla Zonca 34; Rosamani 211; Samani 44; Vascotto 73), friul. caratèl 'id.' (DESF I 311; NP 103), mugl. čaratiél / karatél 'id.'(DDM 25), venez. caratèlo 'id.' (Boerio 136), bellun. karetèl 'botticella da 20-150 litri' (Tomasi 91), trev. caretèl 'barilotto da 2-3 ettolitri' (Bernardi 164) ovvero it. caratèllo 'botticella più lunga che larga' (DEI I 754). In ultima analisi dal lat.mediev. carratum 'specie di botte'; cfr. anche a. lomb. carera 'botte che si trasporta con carri' (buttis carrāria) (DEI I 754).

\section{e) 9. kə'låunik}

Il termine istr.slov., pur isolato, presuppone il cars. kaláti / prim. kálati 'con un recipiente attingere acqua da una fonte' (Bezlaj II 11; Mende RLS 74; Plet. I 380), sostantivato con l'aggiunta del suffisso slov. in -ik, a sua volta derivato dal triest. ed istr. calar 'calare, abbassare'. Dal lat. calāre o chalāre, a sua volta dal gr. khaláō 'allento' (GDDT 112).

f) 9. bar'lọt

Dal triest. ed istr. (D.) dim. bariloto 'barilotto' (GDDT 58; Rosamani 70), affine, anche semanticamente, al friul. barilòt (DESF I 168; NP 40), bis. barilet (Domini 36), venez. barilòto (Boerio 65) nonché it. barilòtto (DELI 1, 117), dim. di it. barile 'piccola botte di legno' (per l'et. v. 541b). 
1. bən'dima; 2. bən'dima; 3. bən'dimå; 4. bən'dima; 5. bən'dimå; 6. ban'dima;

7. bən'dima; 8. ban'dima; 9. ban'dimå; 10. ban'dima

La voce istr.slov., affine al notr. bendîma 'vendemmmia' (Bezlaj I 17; Plet. I 20) / bandima (Mende RLS 42), deriva dall'istr. (C.) bendema / bandema 'id.' (Rosamani 85; Semi 235) / (C., B., P., V., Mt.) vendema (Cernecca 119; Rosamani 1209; Semi 300) con vari riscontri, anche dal punto di vista semantico, nell'area prossima: cfr. il triest. vendema / vendemia (GDDT 778), friul. vendème (NP 1264 seg.), bis. vendema (Domini 532), mugl. vendéma (DDM 178), bellun. vendéma (Tomasi 206), venez. vendèma (Boerio 784) nonché it. vendémmia (DEI V 4008). Dal lat. vindēmia(m), a sua volta da * vinodēmia(m) 'levata, raccolta', composto da vīnu(m) 'vino' e dēmere 'togliere via' (DELI 5, 1421; REW 9343).

506. Vendemmiare; Q. ALI 3975:

8. bəndi'mirat

L'infinito istr.slov. è, con l'aggiunta del suffisso slov., un adattamento dell'istr. (C.) bendemar 'vendemmmiare' (Rosamani 85 ), variante del triest., istr., bis., mugl., bellun., ven. (anche venez.) vendemar 'id.' (GDDT 778 seg.; Rosamani 1210; Domini 532; DDM 178; Tomasi 206; Durante 645; Boerio 784); cfr. inoltre, sempre dello stesso significato, l'istr. (V.) vendemà (Cernecca 119), friul. vendemâ (NP 1264) ovvero it. vendemmiare (DEI V 4008), a sua volta dal lat. vindemiāre 'id.' (REW 9344).

509. Racimolare; Q. ALI 3978:

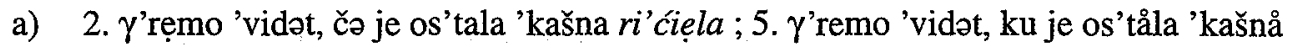
ri'célå (per l'et. v. 469)

b) 3. po'rảplat; 9. po'rảplat / 'rảplat; 10. po'räplat

Il termine istr.slov., affine al cars. rəplət / raplət / räplət / rəpulət 'racimolare' (SDLA-Ts 532) e slov. rapuljáti 'id.' (Plet. II 374), deriva dall'istr.slov. rapolo, a sua volta prestito romanzo.

c) 6. 'rašpət

La voce istr.slov. deriva dall' istr.slov. rašpa, a sua volta prestito romanzo (per l'et. v. $470 \mathrm{a})$.

d) 8. ripi'lirat 
Il termine è d'incerta interpretazione: forse un adattamento dell' istr.slov. rảplat ovvero cars. ropulot 'racimolare' (per l'et. v. 509b), però si tratta puramente di un'ipotesi da accettare con estrema riserva.

510. Sgranellare; Q. ALI 3979:

a) 1. yromo'lerat

Il termine istr.slov. è un adattamento dell' istr. (Mt.) gramolar 'impastare' (Rosamani 450) / (D.) gramolà (Dalla Zonca 146) che concorda con il friul. gramolâ 'gramolare, maciullare (del lino o canapa)' (NP 399), bis. gramular 'maciullare, triturare' (Domini 210), venez., rover. gramolàr 'masticare' (Boerio 313; Prati EV 78), ver. 乌gramolar 'sgranocchiare, sgretolare' (Prati ibid.), ven. (s)gramolare 'masticare, mangiare' (Durante 210), trev. sgramoeàr 'sgranocchiare' (Bernardi 227) ovvero it. gramolàre 'maciullare', di etimologia incerta e probabile relitto mediterraneo (DEI III 1856). Le ipotesi possibili sono inoltre: dai suoni imitativi * cram / * gram, dal lat. carminäre 'cardare', da una base prelat. * gramula(m) 'strumento per maciullare la canapa' (DELI 2, 515; REW 3838a).

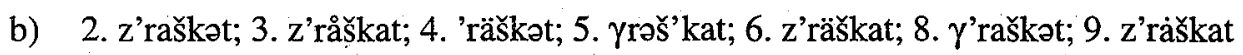

La voce istr.slov. deriva, con l'aggiunta di vari prefissi e suffissi slov., dall' istr.slov. raška / $\gamma$ raška, a sua volta prestito romanzo (per l'et. v. 470b).

\section{c) 5. 'màkinat}

La voce istr.slov. è, attraverso l'aggiunta del suffisso slov., un adattamento dell'istr. (V.) machinà 'trebbiare' (Cernecca 61) che converge, anche semanticamente, con friul. machinâ (NP 544) e padov. (Montagnana) machinar (GDDT 343 sotto la voce machinar). (Per l'et. v. 520b).

d) 10. diž $\gamma r a ' n i r a t$

Adattamento del triest., mugg. e bis. diggranar 'sgranare' (GDDT 205; Rosamani 316; Domini 154) che converge, anche semanticamente, con istr. (C., P1.) decgranar / (R.) de ̧garnâ (Rosamani 300) / (R.) daఢ̧garnâ (Rosamani 290) / (D.) de ̧granâ (Rosamani 300), friul. disgranâ (DESF II 610; NP 249), bis. deçgranar (Domini 144), venez. desgranàr / desgranelàr (Boerio 230), it.ant. disgranare / disgranellare (DEI II 1338) ed it. sgranàre, composto parasintetico con prefisso s- di it. gràno (DELI 5 , 1197), a sua volta dal lat. grānu(m) 'grano' (DELI 2, 516 seg.; REW 3846). 
512. Caricare (l'uva sul carro); Q. RC:

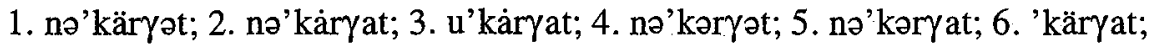

7. 'kảryàt; 8. nə'kəryat; 10. 'kəryat

La voce istr.slov., con l'aggiunta del tipico prefisso e suffisso verbale slov., che si ricollega anche allo slov. (notr.) kárgati, kárgam 'caricare' (Bezlaj II 19), è un prestito rom.: dall' istr. (Ch.) mar. cargar 'imbarcare' (Rosamani 174) riconducibile al venez. cargàr 'caricare' (Boerio 138) ed it.sett. cargare, car(r)egare 'id.' (DEI I 767; Durante 81), dal lat. tardo carricare, a sua volta da carrus 'carro' (REW 1719). Cfr. anche le varianti: istr. (C.) caregàr 'caricare' (Semi 243), triest. carigar 'id.' (GDDT 132), friul. cargâ 'id.' (DESF I 312; NP 104).

513. Scaricare (l'uva dal carro); Q. RC:

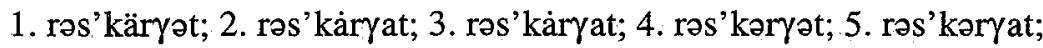

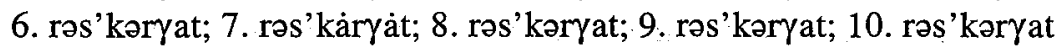
(per l'et. v. 512)

515. Mosto (succo che si spreme dalle uve); Q. ALI 3982:

1. 'mọšt; 2. 'munošt; 3. 'måst; 4. 'mọšt; 5. 'mọšt; 6. 'måst; 7. 'mọšt; 8. 'mast;

9. 'mọšt; 10. 'munäšt

Per il termine istr.slov., affine anche allo slov. mòšt 'vino dolce' (Bezlaj II 196; Mende RLS 96; Plet. I 604), per il quale il Bezlaj propone un'irradiazione dal mated. most 'vino novello' (Bezlaj ibid.), non è esclusa la derivazione dal triest. ed istr. mosto 'id.' (GDDT 390; Cernecca 68; Rosamani 654) / (R.) móusto (Rosamani 655) che ha varie concordanze: cfr., dello stesso significato, il friul. mòst (NP 620), bis. e mugl. most (Domini 288; DDM 100), bellun. móst (Tomasi 125), venez. mosto (Boerio 429) nonché it. mósto (DEI IV 2518). Dal lat. mŭstu(m) di origine sconosciuta, propriamente 'nuovo, recente', agg. applicato soprattutto al vinum, poi sostantivato con valore autonomo (DELI 3, 780; REW 5783).

519. Torchiare l'uva; Q. RC:

- 2. št'rukat; 4. št'rukət; 7. št'rukàt

- 3. štru'kirat; 8. štru'kirat; 10. štru'kirat

Gli infiniti istr.slov. con tipici suffissi sloveni sono degli adattamenti del triest. ed istr. (C.) strucar 'premere; spremere' (GDDT 699; Semi 295) / (Pir., D.) strucâ (Rosamani 1110) con vari raffronti nell' area marginale veneta (per l'et. v. 477). 
520. Pigiatrice; Q. RC:

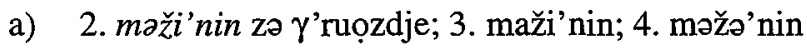

Voce mutuata dal triest. ed istr. (Is., P., Pg., Cf., V., F., O., Ch.) maçinìn 'macinino' (GDDT 366; Cernecca 64; Rosamani 603 seg.; Samani 102; Vascotto 169) / (C., Is.) masenìn (Semi 267; Vascotto ibid.) che concordano, anche semanticamente, con bis. maģinin / maşenin (Domini 273), ven. (anche venez.) maçenín (Boerio 403; Durante 288; Prati EV 99), vic. maçenín / maçinín, valsug., bellun. maģnín, rover. maçním (Prati ibid.), ven.dalm. maçinìn (Miotto 119) ovvero it. macinìno (DEI III 2301), diminutivo di it. màcina (DEI III 2300). Dal lat.parl. * mācina(m) per il class. māchina(m) (DELI 3, 696).

b) 1. 'makińa zə m'let $\gamma$ 'ruzje; 5. 'måkińå zə m'làt $\gamma$ ' ruzjá; 6. 'måkińa od $\gamma$ 'ruzdja zə m'let; 7. 'makińa zə m'let $\gamma$ 'ruzjä; 8. 'makińa zə m'let $\gamma$ 'rọzja

Il termine istr.slov. makińa è la forma palatalizzata della voce it. màcchina (DEI III 2298), attestata anche nel triest. màchina 'macchina (in senso generico)' (GDDT 343) ed istr. màchina 'id.', a Rovigno e Valle anche nel significato di 'trebbiatrice' (Rosamani 561; Cernecca 61); dal lat. māchina (DEI ibid.). La palatalizzazione del termine istr.slov. è avvenuta probabilmente su influsso del friul. màchigne 'macchina; trebbiatrice' (NP 544).

521. Dado dello strettoio; Q. RC:

a) 1. 'vida; 2. 'vida ot št'ruka; 4. 'vida ot št'ruka; 5. 'vidå; 6. 'vida; 7. 'vida ot št'ruka; 8. 'vida (per l'et. v. 521b)

b) 10. in'vida ot št'ruka

La voce istr.slov. è la forma deverbale di triest. ed istr. (C., F.) invidar 'avvitare' (GDDT 314; Semi 262; Rosamani 512), a sua volta dal triest. ed istr. vida 'vite', che ha varie concordanze nell'area veneta (vic., padov., venez., trev., poles., valsug.) (Boerio 793; Doria GDDT 784; Rosamani 1220; Prati EV 200). Dal lat. vitis 'vite' con metaplasma (DEI V 4071; Doria ibid.).

c) 3. 'mọš ot št'rukå

Dall'istr. (A.) mors 'morsa' (Rosamani 651) / (V.) mórsa (Cernecca 68) / (D.) mòrsa (Dalla Zonca 193) che convergono, anche semanticamente, con mugl. mórsa (DDM 100), venez. morsa (Boerio 427) ovvero it. mòrsa (DEI IV 2512), a sua volta dal lat. mŏrsa, propriamente n. pl. del ptc. pass. mŏrsu(m) del lat.parl. * mŏrdere per il class. mordēre, (DELI 3, 777). 
a) 1. 'žọnta; 2. 'žuọnta; 5. 'žọntå; 6. 'žọnta; 8. 'žọnta

Il termine istr.slov., affine allo slov. žộnta 'vinello' (Mende RLS 121; Plet. II 972) e cars. žunta / žuonta 'id.' (SDLA-Ts 600; Štrekelj, Görz. 432), è mutuato dal triest. ed istr. (C., R.) zonta 'bevanda che si ottiene facendo passare dell' acqua attraverso le vinacce già strette' (GDDT 820; Rosamani 1267) che concorda con friul. zònte 'id.' (NP 1319) nonché, nel significato di 'aggiunta', con bis. zonta (Domini 557), ven. (anche venez.) zonta (Boerio 820; Durante 667), ven.dalm. zònta, da cui cr.dalm. žûnta (Miotto 227), ovvero it. giùnta, ptc.pass. di giungere 'aggiungere, congiungere', a sua volta dal lat. iŭngere 'id.' di radice ie. (DELI 2, 502 seg.).

\section{b) 3. š'kảvec; 4. š'kävəc; 7. š'kavjec; 9. s'kảvec}

La voce istr.slov., affine allo slov. skâvec 'vinello' (Mende RLS 108; Plet. II 484) e cars. škavec (Plet. II 630) / škeviéc (Štrekelj, Görz. 400) / škəvəc (SDLA-Ts 600) 'id.', deriva dall' istr. (C., Is.) scavéso 'vinello' (Rosamani 966; Semi 288; Vascotto 261) / (C.) scaves (Semi ibid.) che concordano con friul. s'ciavez 'vino allungato con acqua' (NP 970), mugl. sčavés 'vinello' (DDM 145), bis. scavéz 'id.' (Domini 414), venez. vin scavezzo 'vino adacquato' (Boerio 623), dal venez. scavezzàr 'rompere, spezzare' (Boerio ibid.), passato anche nell'it. scavezzare 'id.' (DEI V 3380; DELI 5, 1148). Alla base sta l'it.sett. cavézzo 'il capo estremo di una pezza di panno', a sua volta dal lat. capĭtium, lat.mediev. cavizium 'cosa spettante al capo' (DEI II 829; REW 1637).

c) * 1. pi'ćot

La voce istr.slov. è un caso di suffisso rom. aggiunto alla radice slov. piti 'bere'.

525. Posca, bevanda d'acqua e aceto; Q. ALI 3988:

a) 1. təmpə'rańe; 2. təmpə'rańa; 3. təmpə'ranà; 4. təmpə'rańa; 5. təmpə'rańå; 6. tampa'rańa; 8. təm'pəråńa

Il termine istr.slov., contraddistinto dal suffisso slov. tipico per il sostantivo verbale, è mutuato dall' istr. (A.) temperà 'bevanda d'acqua e vino' (Rosamani 1145), a sua volta dall'istr. (A.) temperar 'far bevanda, cioè mescolare con acqua un po' di vino oppure aceto' (Rosamani ibid.) / (Pir., R.) intemperâ 'id.' (Rosamani 508) che concordano, nel significato di 'temperare', con friul. temperâ (NP 1181), bis. tenprar (Domini 504), bellun. tenpràr (Tomasi 197), venez. tempèrar / temparàr, temparàr el vin 'annacquare il vino' (Boerio 741) ed it. temperàre 'mescolare specialmente il vino con l'acqua' (DEI V 3745 seg.). Dal lat. temperāre che viene collegato comunemente con tĕmpus 'tempo', anche se ci sfugge il legame semantico (DELI 5, 1323). 
b) 7. 'žọnta; 9. 'žuọntå; 10. 'žọnta (per l'et. v. 524a)

\section{Spina; Q. ALI 3991:}

1. špina; 2. špina; 3. špinå; 4. špina; 6. špina; 7. špina; 8. špina

La voce istr.slov., affine al cars. špína 'spina, zipolo della cannella' (Mende RLS 114; Plet. II 642; SDLA-Ts 612), deriva dal triest. ed istr. (Is., F.) spina 'id.' (GDDT 66; Rosamani 1072; Samani 183; Vascotto 296) che converge, anche semanticamente, con friul. spìne (NP 1093), mugl. e bis. spina (DDM 159; Domini 465), ven.dalm. spìna (Miotto 198), venez. spinàr 'spillare una botte' (Boerio 691) ed it. spìna 'cannella della botte; zaffo della botte', a sua volta dal lat. spina(m) 'formazione vegetale, aculeo' di etimologia incerta: si pensa ad un accomodamento a spīca 'spiga' (DEI V 3592; DELI 5,1253 ).

529. La botte regge, tiene; Q. ALI 3992:

a) 3. je š'tán ; 5. je š'tånå (lod'ricå); 7. je š'táń; 8. je š'tåń; 9. št'åń; 10. je š'tań

Dal triest. ed istr. (C., Is., V., F.) agg. stagno 'forte, saldo' (GDDT 677; Cernecca 108; Rosamani 1086 seg.; Samani 185; Semi 294; Vascotto 299), deverbale da triest. ed istr. stagnar 'stagnare' (GDDT ibid.; Rosamani ibid.), voce di area ven.: cfr., dello stesso significato, le forme deverbali ed i corrispondenti verbi friul. stagn < stagnâ (NP 1107), bis. stagno < stagnar (Domini 471), ven. stagno < stagnar(e) (Boerio 699; Durante 572) ed it. stàgno < stagnàre (DEI V 3614). Dal lat. stāgnu(m), ritenuto comunemente di origine gallica (DELI 5, 1264).

b) 6. je zəštə’’nån

Il ptc. pass. istr.slov., con aggiunta del tipico suffisso slov., è di origine rom. (per l'et. v. 529a).

532. Decotto di mele cotogne e di foglie di pesco usate per profumare le botti; Q. RC:

1. (nəp'ravət) b'rọm; 2. b'rump; 3. b'rọp; 4. (nər'dimo) b'rum; 5 . b'rọm; 6. (nər'dit an) b'rump; 7. (nər'dimo) b'rọm; 8. b'rump; 9. b'ruọn; 10. b'rəmp

I termini istr.slov., affini al cars. bromp / brumba 'lavacro delle botti con acqua bollente' (SDLA-Ts 601), derivano dall' istr. (C., Is., Pir., D., Mt.) brombo 'pampanata (intruso di mele cotogne e di foglie di pesco usate per profumare le botti)' (Dalla Zonca 24; Rosamani 119; Vascotto 60) che converge con triest. bròmbolo 'id.' (GDDT 95), mugl. pl. brónbui 'gorgogli intestinali' (DDM 17), ven. bronba (far la) 'fare la stufa alle botti' (Durante 53), padov. brombar le bote 'id.', venez., ver. brombolàr 'stagnare la botte', padov. brombolare 'gorgogliare' (Prati EV 26), triest. ed istr. brombolar 'id.' (GDDT ibid.; Rosamani 119 seg.) / (Pir., Pl.) brombolâ 'brontolare; far bollicine' (Ive 
171, Rosamani 119), venez. bròmbolo 'pampanata' (Boerio 102), trev. brombo / brombolo 'id.', bellun. brómbol 'id.' (Prati ibid.), ven. dalm. bròmbola 'bolla d'aria' (Miotto 35). I termini citati sono tutti di origine onomatopeica, da un tema bomb-che si riscontra anche nell'it.ant. bombare 'bere vino' (DEI I 558; Prati EV 82 sotto la voce imbombare) e lomb. bombo 'bevanda' (DEI I 557; Prati ibid.).

533. Scemare, calare (l'abbassarsi del vino nella botte); Q. ALI 3995:

a) 1. ka'lavət; 3. kə'låvat; 4. kə'låvət; 5. kə’låva (3.sing.); 6. kə'låvat; 9. kə'låvat (per l'et. v. 533b)

b) 2. 'kàlat; 7. 'kảlàt; 8. 'kảlat

Il termine istr.slov., affine allo slov.dial.(Carso) kaláti / (Primorska) kálati 'abbassarsi' (Bezlaj II 11; Mende RLS 74; Plet. I 380), è mutuato dal triest. ed istr. calar 'calare, abbassare' (GDDT 112; Rosamani 144) che concorda, anche semanticamente, con venez. e ven.dalm. calàr (Boerio 117; Miotto 40), friul. calâ (DESF I 295; NP 91 seg.), mugl. * kalár / čalár (DDM 68 e 21), bis. calar (Domini 73 seg.) ed it. calare (DEI I 669). Dal lat. calāre 'lasciar cadere', a sua volta dal gr. $\chi$ aláō 'allento' (REW 1487).

535. Cantina; Q. ALI 1832:

a) 1. 'kanova; 2. 'kaneva; 3. 'kånevå; 4. 'kåneva; 5. 'kånəvå; 6. 'kåneva; 7. 'kåneva;

8. 'kaneva 9. 'kånevå; 10. 'kåneva

La voce istr.slov., che va con cars. kànova / kànava 'cantina', deriva dal triest. ed istr. (C., P., A.) càneva 'id.' (GDDT 122; Rosamani 160; Semi 242). Cfr. anche, dello stesso significato, triest. ed istr. (C., Is., Pt.) cànova (GDDT 123; Rosamani 162; Vascotto 71)/ (V.) cànua (Cernecca 28) / (R., D.) cànuva (Rosamani 163), friul. ciànive (DESF II 350 seg.; NP 131) / ciàvine (NP 1350), mugl. čánua (DDM 24), bis. càneva (Domini 80), trev., ven., ven.dalm. càneva (Bernardi 157; Boerio 128; Durante 72; Miotto 43; Prati EV 35), bellun. kàneva (Tomasi 87) ed it. cànova (DEI I 725). Dal lat. canăba 'cantina' (REW 1566).

b) 10. kan'tina

Prestito dal triest. ed istr. cantina 'cantina' (GDDT 124; Rosamani 162), voce centro-sett. Cfr., con lo stesso significato, venez. cantìna (Boerio 130), friul. cantìne (DESF I 305; NP 98) ed it. cantìna di etimologia incerta (DEI I 728): probabilmente da it. canto(ne) 'luogo appartato, ripostiglio', dal lat. tardo cănthu(m), a sua volta dal gr. kanthós 'angolo dell'occhio' (DELI 1, 197). 
537. Fondata, feccia; Q. ALI 3998:

1. 'fẹce (pl.); 2. 'fiẹča; 3. 'fẹcå; 4. 'fẹća; 5. 'fečå; 6. 'fẹča; 7. 'feća; 8. 'feca; 9. 'fẹci (pl.); 10. 'fece (pl.)

Il termine istr.slov., affine al cars. féca 'lievito' (Bezlaj I 128; Mende RLS 62), deriva dal triest. ed istr. (A.) feza 1) 'feccia', 2) 'lievito di birra' (GDDT 230; Rosamani 370) / (Is., V.) fèsa (Cernecca 46; Vascotto 108) / (C.) fessa (Semi 253) / (R.) fiesa (Rosamani 374 )/ (Pir.) fieza (Rosamani ibid.), voce di area ven.: cfr. vic., padov., poles., venez., bellun., rover. fezza 'feccia' (Prati EV 63), friul. fèzze (la fèzze dal vin) 'id.' (NP 308 seg.), mugl. fiésa / fésa 'lievito' (DDM 43), che convergono con it. fèccia, dal lat.pop. * fāecea(m), a sua volta da fāex, fāecis 'feccia', relitto mediterraneo (DEI III 1635; DELI 2, 422).

539. Botte; Q. ALI 6102:

a) 1. lud'rica; 2. lod'rica; 3. lod'ricå; 4. lod'rica; 5. lod'ricå; 6. lod'rica; 7. lud'rica; 8 . lod'rica; 9. lod'ricå

La voce istr.slov., affine al cars. e bkr. lodríca 'botte, brenta, otre, recipiente per il vino' (Bezlaj II1 47; Mende RLS 89) nonché scr. lòdrica / ludrìca (Skok II 313), è, con l'aggiunta del suffisso dim. slov. -ica, un adattamento del triest., istr. (Ch., A., Lgr., Lp.) e venez. ludro 'otre' (GDDT 337; Rosamani 553; Boerio 376; Prati EV 195) / padov. udro (Prati ibid.). Dal lat.. ŭtere(m) 'otre' (REW 9102). Nei termini ven. è da notare l'articolo concresciuto (GDDT ibid.).

b) 7. 'kàrətu; 8. 'kärato; 10. 'kärato (per l'et. v. 502d)

c) 10. 'båčva

Il termine istr.slov., citato già dal Pleteršnik sotto la voce bâčva 'botte' (Plet. I 9), che va anche con lo slov. bečvầ 'botte, mastello' (Bezlaj I 15; Plet. I 15), è mutuato dal scr. bačva 'botte' (Skok I 86), a sua volta derivato dall' antica voce psl. dotta * bŭči che, nonostante le difficoltà fonetiche, sembra risalire al lat. tardo buttis, *bŭttia 'botte' (Bezlaj ibid.; REW 1425; Rocchi 1425; Skok ibid.), per cui si tratterebbe di un antico prestito romanzo.

540. Caratello; Q. ALI 6103:

a) 1. 'kärto; 2. 'kärəto; 3. 'kärato; 4. 'kärəto; 5. 'kảrto; 6. 'karatu; 9. 'kärato (per l'et. v. 502d)

b) 6. kəra'tẹlić; 7. kər'tẹlić; 8. kara'tiẹlčić; 10. kara'teunčić 
Formazione istr.slov. dim. di origine rom. (per l'et. v. 502d)

541. Barile; Q. ALI 6104:

a) 1. bə'rilca; 2 . bə'rilca; 3. bà'rilcå; 4. bə'rilca; 5. bə'rilcå; 6. bə'rilca; 7. bə'rilca;

8. ba'rilca; 9 . ba'rilcå

La voce istr.slov., affine al bkr. barîla / barigla 'barile' (Mende RLS 43; Plet. I 12) e cars.. bərilca / bəriyəlca 'id.' (SDLA-Ts 605), è, con l'aggiunta del suffisso dim. slov. -ica / -ca, un adattamento del triest. ed istr. (C., Is.) barila 'barile di 56 litri' (GDDT App. 847; Rosamani 70; Semi 236) / (V.) 'barile di legno di 2 o 3 litri' (Cernecca 20) (per l'et. v. 541b).

\section{b) 10. ba'ril}

Il termine istr.slov., che va con bkr. baril 'piccolo barile da 6-15 boccali' (Mende RLS 43; Plet. I 12), deriva dal triest. ed istr. (Is., R., V.) baril 'barile' (GDDT 58; Cernecca 20; Rosamani 70) che concorda, anche semanticamente, con friul. barîl (DESF I 168; NP 40), bis. baril (Domini 36), mugl. baríle (DDM 10), venez. e bellun. baril (Boerio 65; Tomasi 34) nonché it. barìle di etimologia incerta: alcuni lo fanno risalire al long. bara con aggiunta del suffisso -ile (DEI I 441; REW 1038), altri presuppongono un prerom. *barro- 'fango, argilla', però anche questa ipotesi è da accettare con riserva (DELI 1, 117).

542. Bigoncia; Q. ALI 6105:

a) 1. b'rẹnta; 2. b'riẹnta; 3. b'rẹntå; 4. b'rẹnta; 5. b'rẹntå; 6. b'rẹnta; 7. b'rẹnta;

8. b'renta; 9. b'rentå; 10. b'renta

Il termine, citato già dal Pleteršnik (Plet. I 55), è in stretto collegamento con il cars. brienta 'misura con cui si misurano le biade (30 kg)' (SDLA-Ts 530) / brenta 'bigoncia' (SDLA-Ts 608), triest. ed istr. brenta 'recipiente di legno per il trasporto dell'uva' (GDDT 92; Cernecca 24; Rosamani 115 seg.; Samani 37; Semi 239; Vascotto 59). Cfr. anche mugl. brénta 'bigoncia', friul. brènte 'id.' (DESF I 265; NP 74), bellun., trev. brenta 'tino' (Tomasi 43; Bernardi 106), ver. brènta 'id.', trent. brènta 'mastello' (Durante 51; Prati EV 25), it. brènta attraverso il lomb. brenta (Prati ibid.). Si tratta di una voce di provenienza sett., passata nei dialetti alto-ted. ed in alcune lingue slave; dal lat. mediev. brenta, probabilmente di origine mediterranea (Bezlaj I 42; DEI I 595; DELI 1, 164; Mende RLS 50; REW 1285).

b) 1. b'rentəč; 4. b'räntəć

Il termine istr.slov., affine al cars. brentəč / brəntəč 'bigoncia' (SDLA-Ts 608), deriva da brenta (per l'et. v. 542a). 
543. Mastello; Q. ALI 6106:

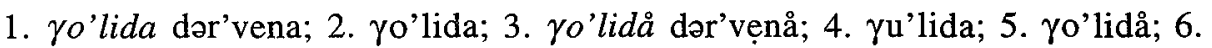

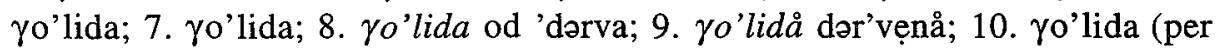
l'et. v. 502b)

544. Bugliolo, bigonciolo; Q. ALI 6107:

a) 8 . bro'kẹt

Dall' istr. (C.) agr. brocheto 'piccolo recipiente per travasare il vino con una doga più lunga che serve da manico' (Rosamani 119), formazione dim. del triest. ed istr. (C., Is., F.) bròca 'brocca' (GDDT 94; Rosamani 118; Samani 38; Semi 239; Vascotto 59) che concorda, anche semanticamente, con bis. broca / dim. brocheta (Domini 61), friul. bròcie / brùcie (NP 77), venez. broca (Boerio 100), trev. bròca (Bernardi 117) nonché it. bròcca di etimo incerto: non improbabile un lat.parl. * brŏcca(m) 'utensile col becco' (brŏccus), influenzato dal gr. brochìs 'calamaio' (o meglio próchus) 'recipiente per versare acqua' (DEI I, 604; DELI 1, 168).

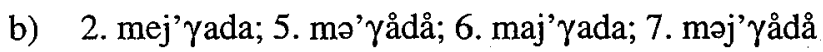

Il termine istr.slov., sebbene isolato, è probabilmente un romanismo: poichè designa il bugliolo a un manico è da supporre la derivazione dal lat. * manicata, a sua volta dal lat. manicus 'manico', cfr. anche it. manico 'id.' (REW 5303a).

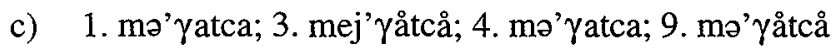

La voce istr.slov., con l'aggiunta del suffisso slov. dim. -ca, è un probabile romanismo (per l'et. v. 544b).

545. Tubo di plastica o di gomma; Q. RC:

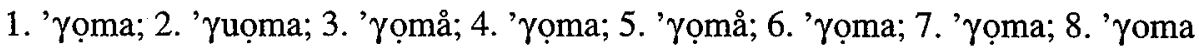

Dal triest. ed istr. (C., Is., B., V.) gòma 'gomma' (GDDT 274; Cernecca 53; Rosamani 445; Vascotto 128) che converge con friul. gòme (NP 395), bis. goma (Domini 209), venez. goma (Boerio 311) ed it. gómma, a sua volta dal lat. tardo gum(m)a per cŭmmi e più tardi gŭmmi. Dal gr. kómmi di origine egiziana (DEI III 1842; DELI $2,510)$.

546. Colare, passare al setaccio; Q. ALI 4346:

1. š'kolət; 2. pərš'kunolat; 3. s’kọlat / prəš'kọlat; 4. pərš'kọlət; 5. pərš'kọlat;

6. pərš'kọlat; 7. pərš'kọlät; 8. prəš'kọlat; 9. pərš'kọlat 
Dal triest. ed istr. (C., F.) scolar 'scolare' (GDDT 596; Rosamani 977; Samani 166; Semi 288) / (V.) scolà (Cernecca 98) che convergono, anche semanticamente, con bis. scolar (Domini 419), friul. scolâ (NP 980), bellun. skolàr (Tomasi 178), venez. scolàr (Boerio 629), ven. scolare (Durante 490) nonché it. scolàre, intensivo di it. colare 'id.' (DELI 5, 1161); cfr. anche lat.mediev. scolāre (DEI V 3407). Dal lat. colāre 'filtrare', a sua volta da cōlum 'filtro' di etimologia incerta (DELI 1, 250 seg.).

547. Fiasco; Q. ALI 796:

a) 1.dəm'jana p'letna; 7. dəm'jana

Dall' istr. (C., B., V.) damiàna 'damigiana' (Cernecca 38; Rosamani 287) / (indf.) domiana (Rosamani 326) / (A.) tomiana (Rosamani 1162) / (C.) damegiana (Rosamani 287) / (indf.) damigiana (Rosamani ibid.) che vanno, anche semanticamente, con bis. damiana (Domini 135), friul. damigiàne / dumiliàne (DESF II 572; NP 224), ven. damigiàna (Durante 131), venez. damegiàna (Boerio 217), ven.dalm. dameana (Miotto 64), bellun. dameiana (Tomasi 49), trev. damiliàna (Bernardi 183) ed it. damigiàna, per etimologia popolare dal fr. dame-jeanne 'signora Giovanna', ma di etimo incerto: si suppone che il punto di partenza sia il prov. damajano da demeg 'metà' (DEI II 1207; DELI 2, 310).

b) 3. k'jåntå; 4. k'janta; 6. k'janta

Il termine istr.slov., nonché cars., è un adattamento dell'it. chiànti 'vino rosso, prodotto nella zona omonima (Chianti) della Toscana' (DEI II 892; DELI 1, 230). Poiché questo vino veniva immesso sul mercato nella tipica bottiglia rivestita di paglia è da ascrivere a ciò il passaggio semantico della voce istr.slov. e carsica.

c) 5. 'bọcå p'lätənå; 2. p'làtəna 'buọca ; 8. 'bọca sp'làtəna; 9. sp'làtənå 'buọcå ; 10. 'boca p'lätəna

Il termine istr.slov., affine al bkr. boca 'boccale, misura per liquidi' (Bezlaj I 29; Mende RLS 47; Plet. I 37) / res. boča (Mende ibid.), deriva dal triest. ed istr. (F.) boza 'bottiglia' (GDDT 89 e 859; Rosamani 110; Samani 37) / (C., Is., V.) bosa (Cernecca 24; Semi 238; Vascotto 57), voce di area ven.: cfr., dello stesso significato, venez. bozza (Boerio 96), ven. e trev. bossa (Bernardi 107; Durante 47), bellun. bòza (Tomasi 42), bis. boza (Domini 58), friul. bòzze 'vecchia misura di mezzo boccale; boccia o bottiglia di vetro bianco' (DESF I 257; NP 70) che vanno con it. bòccia 'bottiglia di forma tozza', dal lat.volg. * bokkja / *bŏttia 'oggetto rotondo' di origine oscura (DEI I 547; DELI 1, 150 seg.; REW 1191). 
548. Damigiana; Q. ALI 1785:

a) 1. dəm'jana; 2. dəm'jana; 3. dəm'jånå; 4. dəm'jana; 5. dam'jånå; 6. dam'jana;

7. dəm'jana (per l'et. v. 547a)

b) 8. flaš'kọn; 10. fraš'kọn

La voce istr.slov., affine al goriz. flaškun 'bottiglione' (Mende RLS 63; Štrekelj, Görz. 398), è probabilmente mutuata dal friul. flas'ciòn 'id.', accrescitivo di flàs'cie 'grossa bottiglia' (NP 323; per l'et. v. 548c), però non è nemmeno da escludere la possibilità che derivi, con l'aggiunta del suffisso rom., dal cars. flaška 'bottiglia' (Bezlaj I 130) che tuttavia non si riscontra nelle parlate istr.slov.

c) 6. fjaš'kọn

Dal triest. fiascon 'damigiana; bottiglione' (GDDT 231; Rosamani 372) che concorda, anche semanticamente, con it. fiascone ed è accrescitivo rispettivamente del triest. ed it. fiàsco 'bottiglia' (cfr. lat. tardo flasco, -onis e flasca), prestito dal got. flask $\overline{0}$ (DEI III 1630; DELI 2, 429).

d) 9. bo'cuọn

Dall'istr. (F., Ch., Lgr.) bozzon 'bottiglione' (Rosamani 111; Samani 37) / (B.) boson (Rosamani 108), accrescitivo di boza / bosa (per 1'et. v. 547c) e voce di area ven.: cfr. (dello stesso significato) ven. bossón (Durante 47), venez. bozzòn (Boerio 96), ven.dalm. bozòn, da cui cr.dalm. bocun (Miotto 31), friul. bozzòn (NP 70).

549. Bottiglia; Q. ALI 795:

1. 'bọca 2. 'buọca 3. 'bọcå; 4. 'bọca; 5. 'bọcå; 6. 'bọca; 7. 'bọca; 8. 'bọca;

9. 'buọcå; 10. 'bọca (per l'et. v. 547c)

550. Turacciolo di sughero; Q. ALI 800:

9. 'šur

Dal triest. ed istr. (C., Is., V., Pl., Z.) suro 'sughero' (GDDT 707; Cernecca 111; Rosamani 1123; Semi 295; Vascotto 309) / (Lgr., Ch.) suvro (Rosamani 1125) / (R.) sóuro (Rosamani 1059) che convergono, anche semanticamente, con friul. sûr (NP 1151), mugl. sur (DDM 165), bis. suro (Domini 489), vic.ant., padov., poles., ven. dalm., venez., trev., bellun. suro (Boerio 724; Miotto 204; Prati EV 183; Tomasi 194), padov., valsug. zuro, vic. surlo, rover. subro, trent. sür, ver. sóaro / sóvaro (Prati ibid.) nonché it. sùghero (DELI 5, 1296). Dal lat. sūbere(m) (con cambio di declinazione), di origine sconosciuta (DELI ibid.) o forse voce del sostrato mediterraneo (DEI V 3674). 


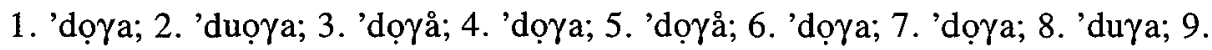
'duộå; 10. 'dọya

Il termine istr.slov., affine allo slov. dóga 'doga della botte o del mastello' (Bezlaj I 106; Mende RLS 59; Plet. I 146), deriva dal triest. ed istr. (V., B., F., Z.) doga 'doga' (Rosamani 324; Samani 61) / (R.) dóuga (Rosamani 329) che concordano, anche semanticamente, con bis. doga (Domini App. II 573), friul. dòghe (DESF II 631; NP 262) / dòve (DESF II 641), bellun. dóga (Tomasi 53), ven.dalm. dòga (Miotto 71), ven. dóa (anche venez.) / dóe / dóva / doga / zova (Boerio 242; Durante 149; Prati EV 58) nonché it. dóga di origine incerta: forse da accostare al lat. tardo dōga 'recipiente' (DELI 2, 358; REW 2714). Secondo il Battisti-Alessio potrebbe essere prestito regionale dal gr. dokós 'trave, travicello', incontratosi con lat. tardo dāga 'recipiente, botte', dal gr. doché 'recipiente' (DEI II 1372), ma si tratta di un'ipotesi dubbia (DESF ibid.).

557. Uzzo, pancia della botte; Q. ALI 6112:

3. 'kärato s treby' $\chi$ on; 7. u'vit 'kärto (per l'et. v. 502d)

559. Tappo del cocchiume; Q. ALI 6114:

9. š'pincå

Il termine istr.slov. è un romanismo con l'aggiunta del tipico suffisso dim. slov. (per l'et. v. 528).

560. Foro nel fondo, spina; Q. ALI 6115:

a) 1. 'buža; 2. 'byža; 3. 'byžå; 4. 'byža; 5. 'byžå; 6. 'byža; 7. 'byža; 8. 'buža; 9. 'byžå; 10. 'byža ot kara'tẹla

La voce istr.slov., affine allo slov. buža 'grotta, cavità' (Bezlaj I 56; Plet. I 73) e cars. (Mačkovlje, Korošci) byža 'id.' (SDLA-Ts 503), deriva dall'istr. buça (C., Pir.) 'buco' (Rosamani 133; Semi 240), a sua volta dal ven. buça 'buco' (Durante 60; Prati EV 30): cfr.. venez. busa nonché busa de leame 'letamaio' e busa de le strade 'pozza' (Boerio 108), ven.dalm. buģa 'fossa' (Miotto 37), bellun. búsa 'fossa; pozza' (Tomasi 45), trev. busa 'buca; fossa; pozzanghera' (Bernardi 109), triest. buça 'occhiello dei vestiti' (GDDT 102), friul. bùse 'buca' (DESF I 286; NP 84). Non convince l'accostamento, proposto dal Bezlaj, all'it.dial. buggia, bugia 'valigia', it.ant. bolgia, dal gallorom. bulga 'sacco' (Bezlaj I 56). Il ven. buso è infatti connesso con it.ant. bùgio 'forato, vuoto' e rifatto sul pl. buçi, a sua volta dal lat. pl. būci, da una base būca e būcum (DEI I 630; GDDT ibid.). 
b) 9. š’pinå (per l'et. v. 528)

562. Cannella da botte; Q. ALI 6117:

a) 1. kə'nẹla; 2. kə'niẹla; 3. ka'nẹlå; 5. ka'nẹlå; 6. ka'nẹla; 7. kə'nẹla; 8. ka'nẹla; 9. ka'niẹlå (per l'et. v. 464b)

b) 4. š'pina; 6. špina; 8. š’pina; 10. špina (per l'et. v. 528)

563. Zipolo della cannella; Q.ALI 6118:

a) 2. š'pina; 3. š'pinå; 4. š'pina; 6. š'pina ot ka'nẹle; 9. š'pinå (per l'et. v. 528)

b) 7. š'pinca (per l'et. v 559) ot kə'nẹle

c) 1. kə'nẹla; 6. špina ot ka'nẹle ; 7. špinca ot ka'nẹle; 8. 'čàp ot ka'nẹle ; 10. 'čäp ot ka'nẹle (per l'et. v. 464b)

564. Mezzule; Q. ALI 6119:

8. 'dộica

Il termine istr.slov. deriva, con l'aggiunta del suffisso dim. slov., dal triest. ed istr. doga (per l'et. v. 553)

565. Lulla, lunetta della botte; Q. ALI 6120:

4. por'tẹla / špor'tẹla; 5. špor'tẹlå

Dal triest. ed istr. (C., Is., Pir., P., B., V.) portela 'sportello, portella, spioncino' (Cernecca 85; Dalla Zonca 212; GDDT 487; Rosamani 820), voce di area ven.: cfr. ven. portèla 'portella' (Boerio 527), ven.dalm. portèl 'boccaporto' (Miotto 160), bis. portela 'portella, sportello' (Domini 352), mugl. * purtiéla 'paratoia' (DDM 130), friul. portèle 'portella' (NP 798). Le voci citate sono forme dim. di porta (friul. puarte).

566. Calastre delle botti; Q. ALI 6121:

a) 2. 'cọkol; 6. 'cọkule (pl.); 9. 'cọkolå za pod'luọžet 'suọt

La voce istr.slov., affine allo slov. cókol 'sandalo' (Bezlaj I 67; Plet. I 86) / cokla 'zoccolo, freno del carro' (Bezlaj ibid.; Mende RLS 53), deriva dal triest. ed istr. (Ch.) zòcolo 'zoccolo' (GDDT 818; Rosamani 1264) / (C.) sòcolo (Rosamani 1047; Semi 292) / (P.) sòcula (Rosamani 1047) / (R.) suòculo (Rosamani 1121) che vanno, anche 
semanticamente, con bis. e friul. zòcul (Domini 557; NP 1316), bellun. zòkola (Tomasi 215), ven. zòcola (Durante 665), venez. zòcolo (Boerio 814) ed it. zòccolo. Dal dim.lat. sŏculu(m), a sua volta da sŏccu(m), prestito penetrato dapprima nel linguaggio teatrale (DEI V 4118; REW 8052). Tuttavia la presenza di z- pone dubbi sulla legittimità di questo etimo. Probabilmente c'è stata una contaminazione con la base * tsŭkko 'ceppo' (F. Crevatin, IL VI 107; DELI 5, 1468).

b) 4. 'kuna; 10. 'kuńa

Dal triest. ed istr. (C., Pir., Is., V., F.) cugno 'cuneo, bietta' (GDDT 191; Cernecca 36; Rosamani 276; Samani 57; Semi 248; Vascotto 93) / (C., Pir., Mt., A.) cogno (Rosamani 227) che concordano, anche semanticamente, con bis. cugno (Domini 128), friul. cugn / cùgno (DESF II 544; NP 207), ven. (anche venez.) e ven.dalm. cugno (Boerio 212; Durante 127; Miotto 61) nonché it. cùneo, voce dotta, dal lat. cŭneu(m) di etimologia incerta (DEI II 1190; DELI 1, 305).

567. Travi sotto la botte; Q. ALI 6121-1:

1. p'läńe; 2. p'jảńe; 3. p'jańà; 4. p'jańe; 5. p'láná; 6. p'jańe; 7. p'lànȧ; 8. p'låńe; 9. p'jańà; 10. p'lańe

Il termine istr.slov., affine al cars. blána /blánja 'trave' (Bezlaj I 24; Mende RLS 46 seg.; Plet. I 32; SDLA-Ts 616) / (Korošci) plańe (SDLA-Ts ibid.), deriva dall'istr. (C., Is., P., B.) piagna 'trave per sostenere le botti' (Rosamani 776; Vascotto 210) che concorda, anche semanticamente, con bis. blagna (Domini 50), mugl. pl. pláńi (DDM 122), friul. plàgne 'striscia di terra tra due filari di viti' (NP 773), bellun. piana 'grossa trave squadrata' (Tomasi 142), ven. (anche venez.) piana 'bietta' (Boerio 502; Durante 374). Se vi accostiamo anche l'emil. piagna 'tegola' ovvero il lat. mediev. plagna 'piastra di legno o di pietra per tegola', l'origine ultima della voce sarebbe il lat. * plānia, a sua volta da plānu(m) 'piano' di origine ie. (DEI IV 2891; DELI 4, 919). Il Zamboni (Zamboni, Romanismi 147) per il cars. blańa e bis. blagna propone invece la derivazione da * filānea (:filu, REW 3306) e li collega al lomb. firańa 'filare di viti', friul. filàine, -gne 'spago' ecc.

569. Bottaio; Q. ALI 5975:

a) 1. bo'tẹr; 2 . bo'tiẹr; 4. bo'ter; 5 . bu'ter; 6 . bo'ter; 7. bu'ter; 8 . bo'ter

La voce istr.slov. che va anche con cars. (Medeazza) butier / (S. Barbara) boter 'bottaio' (SDLA-Ts 615; Zamboni, Romanismi 147) è mutuata dal triest. ed istr. (Is., V., F.) botèr 'id.' (GDDT 88; Cernecca 24; Rosamani 109; Samani 36; Vascotto 57) / (R.) butier (Rosamani 136) a cui si può affiancare, anche semanticamente, il bis., trev., venez., ven.dalm. botèr (Boerio 95; Domini 57; Durante 47; Miotto 31) nonché it. bot- 
tàio, da it. bótte, a sua volta dal lat.tardo bŭtte(m) di cui si cercano gli antecedenti (DEI I 576; DELI 1, 159; REW 1427).

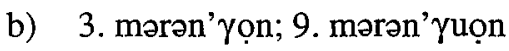

Dal triest. ed istr. (C., Is., V., F., D.) marangon 'falegname' (GDDT 357 seg.; Cernecca 63; Dalla Zonca 188; Rosamani 589; Samani 100; Semi 267; Vascotto 166), voce di ampia area ven.: cfr. ven. (anche venez., bellun. e trev.) marangón 'id.' (Boerio 396; Bernardi 274; Durante 280; Tomasi 116), ven.dalm. marangòn, da cui cr.dalm. marangun (Miotto 113), friul. marangòn / maringòn (NP 566), mugl. e bis. marangón (DDM 93; Domini 268), terg. marangom (Rosamani ibid.) nonché it.ant. marangóne 'cormorano: palombaro; legnaiolo' (DEI III 2359), dal lat.mediev. mergōne(m) per il class. mĕrgu(m) 'smergo' (DELI 3, 718). Recentemente Ch. Schmitt (Sprache und Mensch in Romania, Wiesbaden 1979) ritiene la voce un nome d'agente in -one da * marranca, * marranga (dal lat. * marra 'ascia') 'ascia', quindi marangon 'maestro d'ascia'. Il Doria (GDDT ibid.) scarta questa ipotesi poco persuasiva fino a che non si troverà qualche attestazione del tipo * $\operatorname{mar}(\mathbf{r})$ ancone che testimoni inequivocabilmente il passaggio fonetico piuttosto raro $-\mathbf{n c}->-\mathbf{n g}-$.

\section{c) 10. 'båčvar}

Il termine istr.slov., mutuato dal scr. bàčvar 'bottaio' (Skok I 86), deriva da båčva 'botte', a sua volta antico prestito rom. (per l'et. v. 539c). Il suffisso -ar è di origine lat., recepito comunemente dalle lingue slave tramite mediazione germ. (Vaillant, Gr. comp. IV, 316-320).

572. Botte rigonfia; Q. ALI 6125:

\section{a) 5. 'Yọbəst; 7. 'Yọbảst}

L'agg.istr.slov., affine anche al cars. gobast 'gobbo' (Bezlaj I 154; Plet. I 223), deriva dal triest. ed istr. gobo 'gobbo' (GDDT 273 seg.; Cernecca 53; Rosamani 444), voce di area ven.: cfr., anche semanticamente, il ven. (anche venez.) gobo (Boerio 310; Durante 207), ven.dalm. gòbo (Miotto 90), passato anche nel cr.dalm. (Skok I 583), friul. gobo (NP 393), mugl. góbo (DDM 52) nonché it. gòbbo. Dal lat.volg. * gŭbbus per il classico gibbus 'convesso' (DEI III 1837; DELI 2, 507; REW 3755).

b) 1. imbọm'ban; 8 imbom'bån

Dall'istr. (D., A.) imbombà / imbombado 'inzuppato' (Rosamani 474) / (Is., anche triest.) imbombì (GDDT App. 934; Vascotto 134) che concordano, anche semanticamente, con bis. inbonbì / inbunbì (Domini 219), friul. (im)bòmp (DESF I 244; NP 421), ven. bombo / (im)bombà (Boerio 324; Durante 225; Prati EV 82). Da un tema 
onomatopeico bomb- / bob- indicante il bere (REW 1181) connesso con l'it.ant. bombare 'bere vino' (DEI I 558) e lomb. bombo 'bevanda' (DEI I 557) (v. anche 532).

573. La botte diventa stagna; Q. ALI 6126:

1. je š'tańa (lod'rica); 2. je š'táń ; 3. je š'tań ; 4. je š'tana (lod'rica); 5. je š'tåñå (lod'ricå), štán ('kärto); 6. 'råta štåńa (lod'rica); 7. je š'tán ; 8. je š'tåń ; 9. je štånå (f.); 10. je š'tań (per l'et. v. 529a)

575. Stoppa; Q. ALI 6128:

1. s'tọpa; 2. š'tuọpa; 3. š'tọpå; 4. š'tọpa; 5. štọpå; 6. š'tọpa; 7. š'tọpa; 8. š'tọpa; 9. š'tuọpå

La voce istr.slov., affine al res. štopa 'stoppa' e cars. štǔópa 'id.' (Mende RLS 115; Štrekelj, Görz. 431), deriva dal triest. ed istr. (C., V.) stópa (Cernecca 109; Semi 294) che si accosta, anche semanticamente, al mugl. stópa (DDM 162), friul. stòpe (NP 1121), bellun. stópa (Tomasi 190), ven. (anche venez.) stopa (Boerio 706; Durante 577) ed it. stóppa 'cascame del lino'. Dal lat. stŭppa(m), a sua volta dal gr. styppē di origine ie. (DEI V 3641; DELI 5, 1278).

576. Tartaro, gromma; Q. ALI 6129:

a) 1. $\gamma$ 'ropəda

Dal triest. ed istr. (C., Is., Pir., D.) gròpeda 'gromma' (GDDT 283; Dalla Zonca 148; Rosamani 460; Semi 259; Vascotto 131) che converge, anche semanticamente, con venez.ant. grepola (Boerio 317), vic., padov., venez., ven.dalm., trev., triest. ed istr. grípola (Boerio 318; Durante 213; GDDT 282; Miotto 92; Prati EV 80), bellun. grópola (Tomasi 74), friul. grìpule (NP $406 \mathrm{seg}$.) nonché it.ant. grèppola, voce d' area sett., a sua volta dal lat.mediev. greupum, greupola (DEI III 1869; REW 3875, 3863).

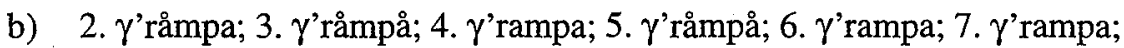

8. $\gamma^{\prime}$ råmpa; 9. $\gamma^{\prime}$ råmpå; 10. $\gamma^{\prime}$ 'rampa

Il termine istr.slov. è connesso con lo slov. grampa 'vinacce; muffa bianca del vino' (Bezlaj I 170; Mende RLS 71; Plet. I 245), cars. grampa / granpa 'gromma' (SDLA-Ts 609) e istr.ciac. grampa 'crusta vinaria in dolio' (Skok I 607). Lo Strekelj (Štrekelj JA XIV 524) propone per il primo la derivazione dall'it. rappa 'crosta', più tardi invece propende per un incrocio tra it. gromma 'muffa del vino' ed it. greppa 'id.' (Štrekelj, Lwk. 21). Lo Skok (Skok ibid.) accosta il termine istr.ciac. all'istr.rom. grapá, gropa 'corrugato'. 
1. 'mufa; 2. 'mufa; 3. 'mufå; 5. 'myfå; 6. 'mufa; 8. 'mufa; 9. 'mufå; 10. 'mufa

Dal triest. ed istr. (V.) mufa 'muffa' (GDDT 391; Cernecca 69; Rosamani 657) / (R., D.) móufa (Rosamani 655) che concordano, anche semanticamente, con mugl. múfa (DDM 101), bis., ven. (anche venez.) mufa (Boerio 431; Domini 290; Durante 314 ), friul. mùfe (NP 625) ed it. mùffa, voce espressiva dalla radice * muff- che sta alla base di parole rom. e germ. fra le quali non è facile stabilire rapporti di priorità (DEI IV 2525; DELI 3, 783 seg.).

578. Aceto; Q. ALI 974:

1. 'vocät; 2. 'uي̃ocảt; 3. 'ọcàt; 4. 'vọcət; 5. 'ọcat; 6. 'ọcat; 7. 'unocảt; 8. 'ự̣cət;

9. 'unọcàt; 10. 'unäcot

Il termine istr.slov., affine anche al pkm. e goriz. ócet 'aceto' (Bezlaj II 239; Plet. I 756), è un antico prestito rom. con mediazione got.: risale infatti, tramite il got. akcit al lat. acētu(m) 'aceto', a sua volta dalla radice ie. * ac- che indica l'essere pungente (DEI I 37; DELI 1, 15; Rocchi 98).

579. Acquavite; Q. ALI 1814:

10. t'råpa

Dal triest. ed istr. (C., Is., P., F.) trapa 'grappa' (GDDT 752 e App. 1017; Rosamani 1170; Samani 201; Semi 298; Vascotto 322) che va, anche semanticamente, con bis. trapa (Domini 516) e ven. tràpa (Durante 629); cfr. anche friul. trape 'vinacce' (NP 1208 seg.) e mugl. trápi 'id.' (DDM 171), dal ted. Treber 'id.' per incontro con grappa (DEI V 3864).

580. Alambicco; Q. RC:

a) 1. ləm'bik; 3. ləm'bik; 4. ləm'bik; 5. lam'bik; 6. lam'bik; 8. ləm'bik

Dal triest. (attestato già dal 1450-1510) ed istr. (Is., V.) lambìco 'alambicco' (Cernecca 57; Rosamani 523; Vascotto 149) / (R.) lambéico (Rosamani ibid.) affine, anche semanticamente, al bis. Ianbic (Domini 242), friul. lambìc (NP 498), bellun. lanbik (Tomasi 107), venez. lambìco (Boerio 358) ed it. lambìcco, voce dotta, dall'ar. anbīq, a sua volta dal gr. ámbix 'tazza' (DEI III 2154; DELI 3, 647).

b) 2. štə'ńada; 5. štə'ńådå; 7. štə'ńåda; 8. š’tånåda; 9. štańådå; 10. šta'ńåda

Dal triest. (anche terg.) ed istr. (Is., Pir., V., D., A.) stagnada 'pentola stagnata, paiolo' (GDDT 677; Cernecca 108; Rosamani 1086; Vascotto 299) che converge, anche dal punto di vista semantico, con bis. stagnada (Domini 471), mugl. stańáda (DDM 
161), friul. stagnàde (NP 1107), bellun. stańada (Tomasi 189), venez. stagnada (Boerio 699), ven. dalm. stagnàda (Miotto 200) ed it. stagnata 'recipiente di stagno', da stagnare, a sua volta dal lat. stagnāre (DEI V 3614).

582. Bicchierino di acquavite; Q. ALI 797-2:

a) 1. biće'rin; 4. biče'rin; 5. bəčə'rin; 6. biče'rin; 8. bićə'rin; 9. bićə'rin; 10. biče'rin

Dal triest. ed istr. (Is., B., F.) bicerin 'bicchierino' (GDDT 71; Rosamani 90; Samani 33; Vascotto 52) / (R.) biciaréin (Rosamani ibid.), dim. di triest. ed istr. bicer 'bicchiere': cfr. bis. biciarin (Domini 47), friul. bicierin (DESF I 214; NP 54), trev. bicerìn (Bernardi 105), ven.dalm. bicerìn, da cui cr.dalm. bićerîn (Miotto 25), ed it. bicchierino, dim. di bicchière. Si contendono l'origine della voce il gr. bîkos 'orcio', il lat.parl. * bicariu(m) 'piccolo orcio' ed il franc. * bikari (DEI I 510; DELI 1, 138).

\section{b) 7. bičə'rinčić}

Alla base del termine istr.slov. c'è il dim. triest. ed istr. bicerin con aggiunta del suffisso dim. slov. -ič (per l'et. v. 582a).

583. Coda della grappa; Q. RC:

a) 1. f'lẹma; 2. f'liẹma; 3. f'lẹmå; 4. p'lẹma

Dal istr. (C.) flema 'flemma; avanzo della distillazione dell'alcool' (Rosamani 385) che converge con friul. flème 'id.' (NP 324), venez. flema 'flemma' nonché it. flèmma 'sostanze chimiche che si formano durante la fermentazione alcoolica del glucosio; lentezza' (DEI III 1666). Dal lat. tardo phlěgma, a sua volta dal gr. phlégma 'infiammazione; catarro', da gr. phlégein 'bruciare' d'origine ie. (DELI 2, 442).

b) 7. škarto

Dal triest., istr. (R.) e venez. scarto 'scarto' (Boerio 621 seg.; Dalla Zonca 229; Rosamani 964); cfr. anche friul. e bis. scart 'id.' (Domini 412; NP 958), it. scarto 'ciò che viene scartato', deverbale da scartare (DEI V 3378; DELI 5, 1147; REW 1866).

c) 8. žon'tẹla

Il termine istr.slov., che si può confrontare con il venez. zontarèla/ zontadina 'piccola aggiunta' (Boerio 821), è il dim. del triest. ed istr. zonta (per l'et. v. 524a).

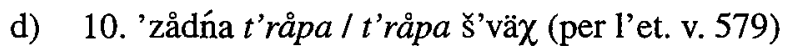




\section{BIBLIOGRAFIA}

$\mathrm{ALI}=$ Atlante Linguistico Italiano, materiali inediti, Torino-Udine.

ASLEF = Atlante Storico-Linguistico-Etnografico del Friuli-Venezia Giulia, ed. G.B.

Pellegrini, Padova 1972-.

Bernardi = U. BERNARDI, Abecedario dei villani, Treviso 1981.

Bezlaj = F. BEZLAJ, Etimološki slovar slovenskega jezika, I (A-J), Ljubljana 1976; II

(K-O), Ljubljana 1982; III (P-S), Ljubljana 1995.

Boerio = G. BOERIO, Dizionario del dialetto veneziano, II ediz., Venezia 1856.

Cernecca $=$ D. CERNECCA, Dizionario del dialetto di Valle d'Istria, Trieste 1986.

Dalla Zonca = G.A. DALLA ZONCA, Vocabolario dignanese-italiano, Trieste 1978.

$\mathrm{DDM}=\mathrm{D}$. ZUDINI - P.P. DORSI, Dizionario del dialetto muglisano, Udine 1981.

DEI = C.BATTISTI-G.ALESSIO, Dizionario etimologico italiano, Firenze 1950-57.

DELI = M. CORTELAZZO-P. ZOLLI, Dizionario etimologico della lingua italiana,

1-5 (A-Z), Bologna 1979-1988.

DESF $=$ G.B. PELLEGRINI - M. CORTELAZZO - A. ZAMBONI, Dizionario eti-

mologico storico friulano, I (A-Ca), II (Ce-Ezzitâ), Udine 1984-.

Domini = S. DOMINI - A. FULIZIO - A. MINIUSSI - G. VITTORI, Vocabolario

fraseologico del dialetto 'bisiàc', Bologna 1985.

Durante = D. DURANTE - GF. TURATO, Dizionario etimologico veneto-italiano,

Padova 1975.

FPF $=$ G.B. PELLEGRINI - A. ZAMBONI, Flora popolare friulana, Udine 1982.

GDDT = M. DORIA - C. NOLIANI, Grande dizionario del dialetto triestino, Trieste 1987.

IVE $=$ A. IVE, I dialetti ladino-veneti dell'Istria, Strassburg 1900.

$\mathrm{JA}=$ Archiv für slavische Philologie. Hg.v.V.Jagic, Berlin 1876-1929.

Mende RLS $=$ M. MENDE, Romanische Lehnwörter im Slowenischen. Inaugural-Dis-

sertation zur Erlangung des Doktorgrades. (Copia dattiloscritta, Slavisches Semi-

nar der Freien Universität Berlin, sign: Fak. No 263.) Berlin b.1.

Miotto II = L. MIOTTO, Vocabolario del dialetto veneto-dalmata, II ediz. riveduta e ampliata, Trieste 1991.

NP = G.A. PIRONA- E. CARLETTI- G.B. CORGNALI, Il nuovo Pirona, Vocabolario friulano, Udine 1977, ristampa dell'ediz. del 1935.

Plet. = A.A. WOLF- M. PLETERŠNIK, Slovensko-nemški slovar, I-II, Ljubljana 18941895.

Prati EV = A. PRATI, Etimologie venete, Venezia-Roma 1968.

REW = W. MEYER-LÜBKE, Romanisches Etimologisches Wörterbuch, III. ediz., Heildelberg 1935.

Rocchi $=$ L. ROCCHI, Latinismi e romanismi antichi nelle lingue slave meridionali, Udine 1990.

Rosamani = E. ROSAMANI, Vocabolario giuliano, Bologna 1958, Trieste 1990 (I ristampa). 
Samani = S. SAMANI, Dizionario del dialetto fiumano, II ediz., Venezia 1980.

SDLA-Ts = R. COSSUTTA, Slovenski dialektološki leksikalni atlas Tržaške pokrajine, Trieste 1987.

Semi = F. SEMI, El parlar s'ceto e neto de Capodistria (testi antichi e moderni, glossario, itinerario grafico, documentazione fotografica), Venezia 1983.

Skok = P. SKOK, Etimologijski rječnik hrvatskoga ili srpskoga jezika, I-IV, Zagreb 1971-74.

Štrekelj, Görz. = K. ŠTREKELJ, Morphologie des Görzer Mittelkarstdialektes. (SBWA CXIII 1.) Wien 1887.

Štrekelj, Lwk. = K. Štrekelj, Zur slavischen Lehnwörterkunde, (DAW L 3.), Wien 1904. TAF $=$ G.B. PELLEGRINI - C. MARCATO, Terminologia agricola friulana, Udine 1988-.

Tomasi = G. TOMASI, Dizionario del bellunese arcaico (in verità del dialetto bellun . rustico di Revine), Belluno 1983.

Vaillant, Gr. comp. = A. VAILLANT, Grammaire comparée des langues slaves, IV, Paris 1974.

Vascotto = A. VASCOTTO, Voci della parlata isolana nella prima metà di questo secolo, Imola 1987.

Zamboni, Romanismi = A. ZAMBONI, Romanismi e altri strati linguistici nella Slavia triestina, Quaderni Patavini di linguistica, Monografie.8 per Giovan Battista Pellegrini, Padova 1991.

\section{ABBREVIAZIONI}
A. $=$ Albona
B. $=$ Buie
C. $=$ Capodistria
Cf. $=$ Canfanaro
Ch. $=$ Cherso
D. = Dignano
F. $=$ Fiume
Gall.= Gallesana
Is. = Isola
Lgr. $=$ Lussingrande
Lp. = Lussinpiccolo
Mt. $=$ Montona
O. = Orsera
P. $=$ Parenzo 


$$
\begin{aligned}
& \text { Pg. = Pinguente } \\
& \text { Pl. = Pola } \\
& \text { Pt. = Portole } \\
& \text { R. = Rovigno } \\
& \text { V. = Valle d'Istria } \\
& \text { Z. = Zara } \\
& \text { bkr. = belokranjsko } \\
& \text { cars. = carsolino } \\
& \text { goriz. = goriziano } \\
& \text { Q. = Questionario } \\
& \text { notr. = notranjsko } \\
& \text { pkm. = prekmursko } \\
& \text { prim. = primorsko } \\
& \text { res. = resiano } \\
& \text { stir.sorient. = stiriano sudorientale (Štajerska) }
\end{aligned}
$$

\section{Povzetek \\ ROMANIZMI V VINOGRADNIŠKI TERMINOLOGIJI SLOVENSKE ISTRE}

Avtorica je iz svoje doktorske disertacije Poljedelska in vinogradniška terminologija v govorih Slovenske Istre, ki jo je zagovarjala 14. januarja 1994 na Filozofski fakulteti v Ljubljani (mentor prof. Tine Logar, somentorica prof. Alenka Šivic-Dular), izbrala in etimološko utemeljila vse tiste termine s področja vinogradništva, ki so po svojem izvoru romanski. V tej panogi so namreč romanizmi trdno zasidrani in že na prvi pogled pričajo o neprestanih stikih istrskih ljudi $z$ romanskim svetom; njihova podrobna analiza pa razgrinja pozitivne podatke, ki kažejo na raznovrstnost, ki nam omogoča jasneje začrtati jezikovno sliko tega ozemlja in različne tokove njegove romanizacije. 